ARTICLE

\title{
An orphan protein of Fusarium graminearum modulates host immunity by mediating proteasomal degradation of TaSnRK1 $\alpha$
}

Cong Jiang (1) 1,2, Ruonan Hei ${ }^{1}$, Yang Yang ${ }^{1}$, Shijie Zhang ${ }^{3}$, Qinhu Wang ${ }^{1}$, Wei Wang ${ }^{1}$, Qiang Zhang ${ }^{1}$, Min Yan $^{1}$, Gengrui Zhu', Panpan Huang ${ }^{1}$, Huiquan Liu (i) ${ }^{1 \times} \&$ Jin-Rong Xu (iD ${ }^{2 凶}$

Fusarium graminearum is a causal agent of Fusarium head blight (FHB) and a deoxynivalenol (DON) producer. In this study, OSP24 is identified as an important virulence factor in systematic characterization of the 50 orphan secreted protein (OSP) genes of $F$. graminearum. Although dispensable for growth and initial penetration, OSP24 is important for infectious growth in wheat rachis tissues. OSP24 is specifically expressed during pathogenesis and its transient expression suppresses BAX- or INF1-induced cell death. Osp24 is translocated into plant cells and two of its 8 cysteine-residues are required for its function. Wheat SNF1-related kinase TaSnRK1 $\alpha$ is identified as an Osp24-interacting protein and shows to be important for $\mathrm{FHB}$ resistance in TaSnRK1 $\alpha$-overexpressing or silencing transgenic plants. Osp24 accelerates the degradation of TaSnRK1 $\alpha$ by facilitating its association with the ubiquitin-26S proteasome. Interestingly, TaSnRK1 $\alpha$ also interacts with TaFROG, an orphan wheat protein induced by DON. TaFROG competes against Osp24 for binding with the same region of TaSnRK $\alpha$ and protects it from degradation. Overexpression of TaFROG stabilizes TaSnRK1 $\alpha$ and increases FHB resistance. Taken together, Osp24 functions as a cytoplasmic effector by competing against TaFROG for binding with TaSnRK1 $\alpha$, demonstrating the counteracting roles of orphan proteins of both host and fungal pathogens during their interactions.

\footnotetext{
${ }^{1}$ State Key Laboratory of Crop Stress Biology for Arid Areas and NWAFU-Purdue Joint Research Center, College of Plant Protection, Northwest A\&F University, 712100 Yangling, Shaanxi, China. ${ }^{2}$ Department of Botany and Plant Pathology, Purdue University, West Lafayette, IN 47907, USA. ${ }^{3}$ School of Life

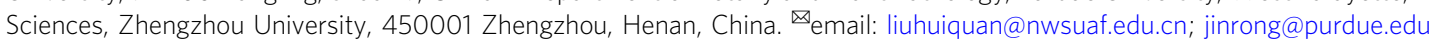


C omparative genome studies have revealed that fungal pathogens have a cadre of orphan genes that are restricted to a single species or narrow clade. Although the vast majority of them are of unknown functions, these taxonomically restricted orphan genes are thought to play important roles in lineage-specific adaptations ${ }^{1-3}$. Plant pathogenic fungi may evolve novel orphan genes to facilitate their infection or enhance virulence. Fungal effectors are good examples of orphan genes that have evolved for plant infection as many of them lack homologs in closely related species. To date, effectors of various molecular mechanisms have been identified and characterized in different fungi ${ }^{4,5}$. Most of them are small secreted proteins that are cysteine-rich but lack a common structural motif. In Magnaporthe oryzae, a model fungal pathogen, whereas apoplastic effectors are usually secreted via the conventional endoplasmic reticulum to Golgi route and accumulated in the apoplast, some cytoplasmic effectors are able to be secreted through the biotrophic interfacial complex (BIC) and translocated into plant cells ${ }^{4,6}$. The targets of fungal effectors also vary significantly in their functions, including transcription factors, protein kinases, and proteins or compounds that are involved in plant defense, signaling, and metabolic pathways ${ }^{7-10}$. For example, the Tin 2 and Cmul effectors of Ustilago maydis target the ZmTTK1 kinase for affecting cell wall lignification and chorismate for affecting the metabolic status of infected plant cell, respectively ${ }^{11-13}$.

Fusarium graminearum is a causal agent of Fusarium head blight (FHB), which is one of the most important diseases of wheat and barley worldwide. It develops compound appressoria and infection cushions for plant penetration ${ }^{14}$. After penetration, invasive hyphae grow inter-cellularity and intra-cellularly in infected plant tissues and develop bulbous, irregular invasive hyphae that are morphologically distinct from epiphytic hyphae ${ }^{15,16}$. Infectious growth then spreads from the initial infection site to neighboring spikelets on the same wheat heads via the rachis, resulting in the blight of entire wheat heads. In the past decade, many genes important for different infection processes have been identified in F. graminearum ${ }^{17-20}$, including the putative FGL1 and FgNahG effector genes. FGL1 encodes a secreted lipase that can release free fatty acids to inhibit innate immunity-related callose formation during wheat head infection $^{21}$. FgNahG is predicted to encode a secreted salicylate hydroxylase that can covert salicylic acid (SA) to catechol. The FgnahG deletion mutant was reduced in virulence and expression of $\mathrm{FgNahG}$ in Arabidopsis reduced its resistance against $F$. graminearum ${ }^{22}$. Recently, a non-ribosomal octapeptide, fusaoctaxin $\mathrm{A}$, was identified as a virulence factor that is required by F. graminearum for cell-to-cell invasion in wheat coleoptiles ${ }^{23}$.

Besides causing severe yield losses, F. graminearum is a producer of the trichothecene mycotoxin deoxynivalenol (DON) 24,25 . As an inhibitor of eukaryotic protein synthesis, DON is also phytotoxic and an important virulence factor. The TRI5 gene, which is essential for DON biosynthesis, is expressed in infection cushions during early stages of infection ${ }^{14}$. The tri5 deletion mutant is defective in spreading from the infected florets to other spikelets on the same head via the rachis ${ }^{26}$ and transgenic plants expressing a UDP-glucotransferase gene that glycosylates DON are increased in resistance against $\mathrm{FHB}^{27}$. Overexpression of TaFROG that encodes a Pooideae-specific orphan protein induced by DON treatment also increased resistance against $F$. graminearum $^{28}$. In wheat, TaFROG interacts with the TaSnRK1a protein kinase $\mathrm{e}^{28}$ and a NAC transcription factor ${ }^{29}$. Although germplasm with complete resistance or immunity to F. graminearum is lacking, more than 50 quantitative trait loci (QTLs) conferring various degrees of FHB resistance have been identified in wheat. To date, only Fhb1, a QTL that confers resistance to pathogen spread but not the initial infection, has been characterized at the molecular level. The candidate Fhbl genes that have been reported include TaPFT encoding a pore-forming toxin-like protein ${ }^{30}$ and TaHRC-R or His ${ }^{R}$ encoding a histidinerich calcium-binding protein ${ }^{31,32}$. However, the underlying mechanism of these candidate Fhb1 genes for FHB resistance remains to be characterized.

The F. graminearum genome is predicted to encode hundreds of orphan genes ${ }^{33}$ but their roles in the pathogenic interaction and co-evolution of F. graminearum with its host plants remain to be characterized. Because small secretory proteins may function as effectors to interfere with plant immunity during infection $^{34-36}$ and no cytoplasmic effectors have been identified in F. graminearum, in this study we systematically characterized all the 50 genes encoding orphan secretory proteins (OSPs) and identified OSP24 as an effector that is specifically expressed during plant infection and important for infectious growth in the rachis tissues of infected wheat heads. Transient expression of Osp24 in plant cells suppressed BAX-induced or INF1-induced program cell death. Furthermore, we identified the SNF1-related kinase TaSnRK1a as an Osp24-interacting protein and showed that TaSnRK1a is important for resistance against F. graminearum by RNA silencing or over-expression in transgenic plants. Osp24 may promote the degradation of TaSnRK1a through the ubiquitin-26S proteasome system ${ }^{37}$. TaFROG competed against Osp24 for binding to the same region of TaSnRK1a. Overexpression of TaFROG in wheat stabilized TaSnRK1a and increased resistance against $F$. graminearum. Overall, results from this study showed that the OSP Osp24 in F. graminearum functions as a cytoplasmic effector targeting TaSnRK1a for degradation but the wheat orphan protein TaFROG competes with Osp24 for binding with TaSnRK1 $\alpha$ and prevents its degradation, indicating the evolving and active adoption of orphan proteins in the arms race between the pathogen and its host.

\section{Results}

Identification and characterization of OSP genes in F. graminearum. Among all the predicted protein-coding genes in the F. graminearum genome, 971 of them $(\sim 7.3 \%)$ were identified as orphan genes using the bioinformatics analyses described in the "Methods" section. On average, these orphan genes encode smaller proteins than genes conserved in other species (Fig. 1a) and tend to be less transcribed or transcribed at lower levels (Fig. 1b) based on published RNA-seq data ${ }^{20,30}$. Fifty of them were predicted to encode proteins with signal peptides (SPs) and named OSP genes in this study. For a number of these OSPs, the mature peptides have fewer than 100 amino acid (aa) residues and are cysteine-rich (Fig. 1c), which is similar to the general characteristics of fungal effector proteins ${ }^{4,5}$.

To determine their functions, gene replacement mutants were generated for the 50 OSP genes by the split-marker approach in the wild-type strain PH-1 (Supplementary Table 1, Supplementary Fig. 1). All the resulting mutants were normal in vegetative growth, conidiation, and sexual reproduction (Fig. 1d; Supplementary Table 2). In wheat head infection assays, most of the mutants were normal in virulence (Supplementary Table 2). However, mutants deleted of three individual OSP genes, OSP24 (FGSG_11564), OSP25 (FGSG_11647), and OSP44 (FGSG_13464), were significantly reduced in virulence in repeated infection assays with wheat heads of cultivar Xiaoyan 22 (Fig. 1e). On average, the disease index of the osp24,osp25, and osp44 deletion mutants was reduced $51 \%$, $37 \%$, and $36 \%$, respectively, in comparison with that of $\mathrm{PH}-1$ (Fig. 1f). The osp24, osp25, and osp44 mutants also were reduced in virulence in infection assays with wheat cultivar Zhoumai $36^{38}$ (Supplementary Fig. 2), indicating that the role of OSP24 in plant infection is not cultivar-specific. When assayed with the inoculated 
a

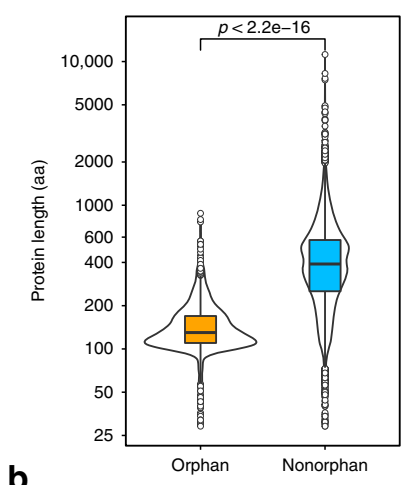

b

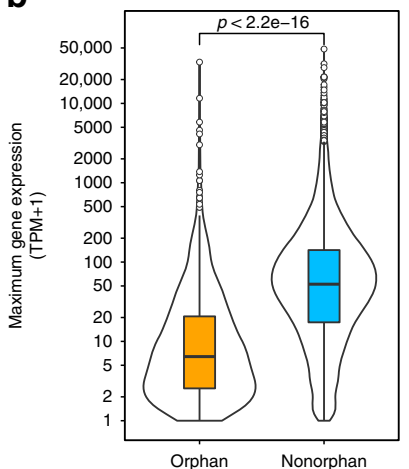

C Protein length

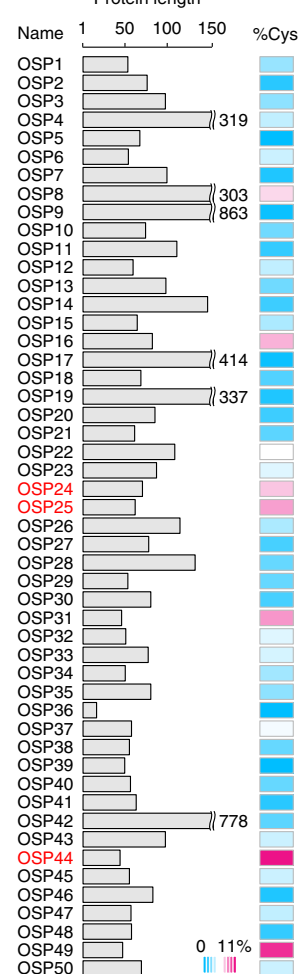

d
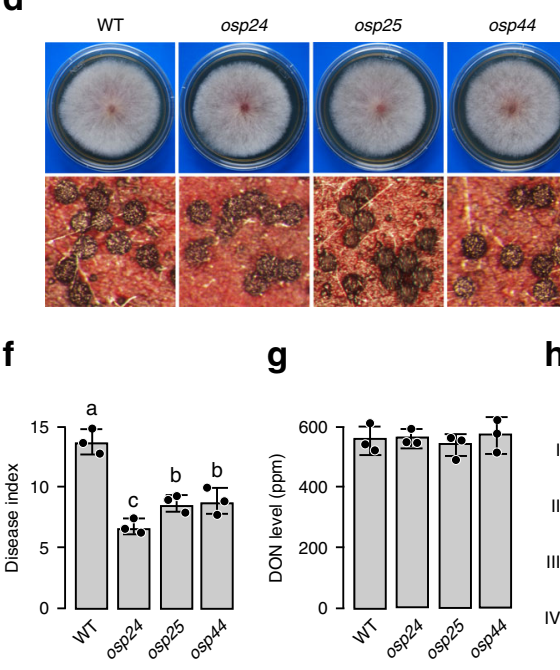

g

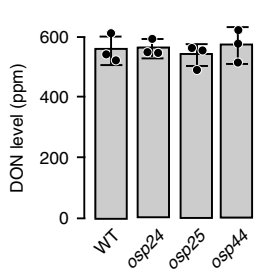

i

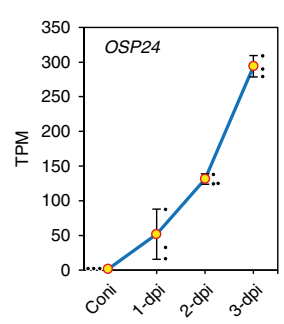

e

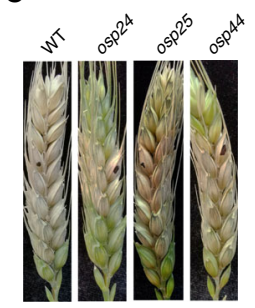

h

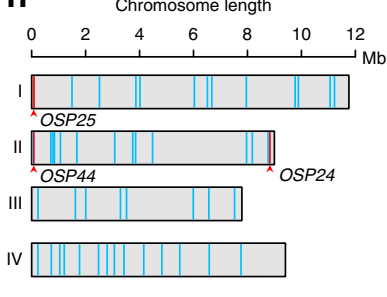

Fig. 1 Characterization of orphan genes encoding secreted proteins in Fusarium graminearum. a Comparative analysis of the length of proteins encoded by orphan and non-orphan genes with the Wilcoxon rank-sum test $(p<2.2 \mathrm{e}-16)$. b Comparative analysis of the expression levels of orphan and nonorphan genes with the Wilcoxon rank-sum test $(p<2.2 \mathrm{e}-16)$. For each gene, the maximum gene expression level was estimated from its transcripts per kilobase million (TPM) in RNA-seq data of conidia, vegetative hyphae, perithecia, and infected wheat heads. c Length and Cys (cysteine) content (\%) of mature orphan secretory proteins (OSPs). The three OSPs important for virulence are in red. d Representative images of 3-day-old PDA cultures and 8-dpf ( 8 days post-fertilization) mating plates of the wild-type strain $\mathrm{PH}-1$ (WT) and the osp24, osp25, and osp44 deletion mutants. e Representative images of wheat heads infected with marked strains were photographed at 14 dpi. $\mathbf{f}$ The disease index of WT and three osp mutants. Error bar represents standard deviation (SD) from mean (marked with black dots on the bars) of three independent experiments $(n=3)$ with at least 10 wheat heads examined in each experiment. Different letters indicate significant differences based on ANOVA analysis followed by Duncan's multiple range test $(P=0.05)$. $\mathbf{g}$ Mean and standard deviations of DON levels in diseased wheat spikelets inoculated with WT or three osp mutants based on data from three biological replicates $(n=$ 3). No significant differences was observed based on ANOVA analysis followed by Duncan's multiple range test $(P=0.05)$. $\mathbf{h}$ Distribution of the OSP genes (blue vertical bars) on the four chromosomes of $F$. graminearum. Red bars and arrowheads indicate the positions of OSP24, OSP25, and OSP44. i Expression levels of the indicated OSP genes based on their TPMs in RNA-seq data of conidia and infected wheat heads ${ }^{20}$ sampled at 1-dpi, 2-dpi, or 3-dpi. Error bar represents SD from three biological replicates $(n=3)$.

spikelets sampled at $14 \mathrm{dpi}$, DON production was normal in these three mutants (Fig. 1g). The osp24 mutant that had the lowest virulence was selected to assay for TRI5 gene expression. In the inoculated spikelets, the transcription level of TRI5 also was similar between the wild type and osp24 mutant (Supplementary Fig. 3). Interestingly, the OSP24, OSP25, and OSP44 genes all are located in the sub-telomeric regions (Fig. 1h), which may facilitate their rapid evolution $^{39}$. Moreover, they were all specifically expressed or significantly up-regulated in infected wheat heads (Fig. 1i; Supplementary Table 3). These results indicate that the secreted Osp24, Osp25, and Osp44 proteins are important for the full virulence of F. graminearum, possibly by functioning as effectors during plant infection.

The osp24 mutant is defective in infectious growth in the rachis tissues. Because the osp24 deletion mutant was most significantly reduced in virulence, we selected it for further characterization. In infection assays with corn silks, tomatoes and Arabidopsis floral tissues, the osp24 mutant was normal in virulence (Supplementary Fig. 4), suggesting a host-specific role of Osp24 during plant infection. To further characterize its function during wheat infection, the osp24 mutant was assayed for the formation of infection cushions and growth of invasive hyphae. When examined by scanning electron microscopy (SEM), abundant infection cushions were observed on wheat lemma inoculated with the osp24 mutant at 2 days post-inoculation (dpi). In comparison with the wild type, the osp24 mutant had no obvious defects in infection cushion formation (Fig. 2a). Deletion of OSP24 also had no obvious effects on the initial plant penetration and development of invasive hyphae in lemma tissues at 2 dpi (Supplementary Fig. 5). However, extensive hyphal growth to the wild-type level was not observed in the rachis tissues of osp24-infected wheat heads at $5 \mathrm{dpi}$ (Fig. 2b). When assayed by qPCR with genomic DNA isolated from infected wheat heads at $5 \mathrm{dpi}$, fungal biomass was significantly reduced in wheat heads inoculated with the osp24 mutant in comparison with those inoculated with $\mathrm{PH}-1$ (Fig. 2c). These results indicate that OSP24 plays an important role in infectious growth and spreading in the wheat rachis tissues.

The SP of Osp24 is required for its secretion and function. The SP-SUC2 construct was generated by cloning the 22-aa SP of 
a

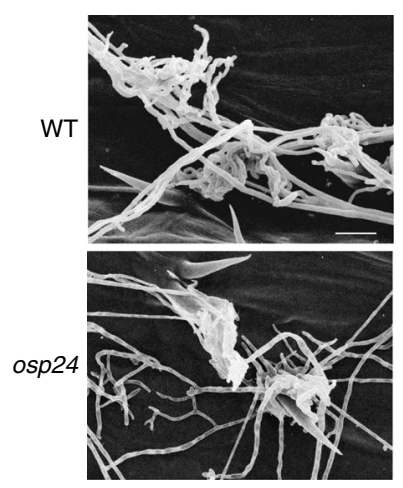

b

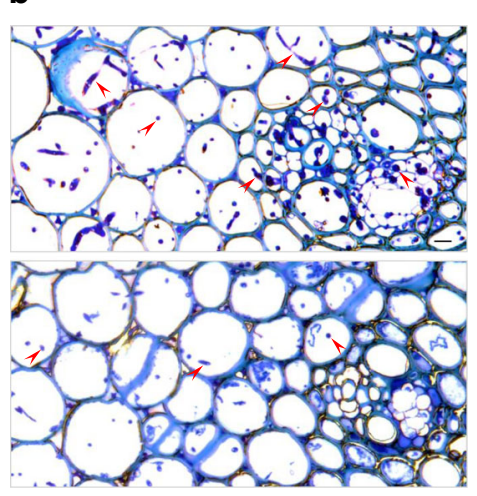

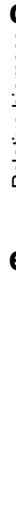

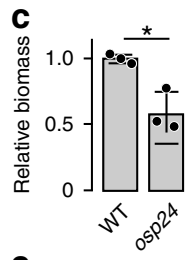

d

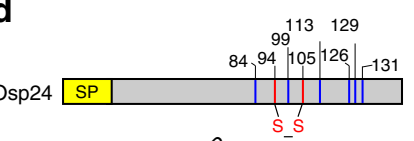

g

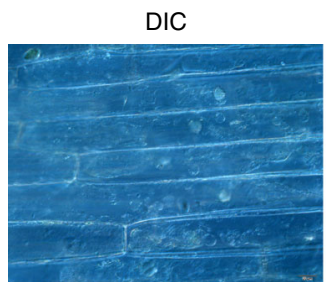

i

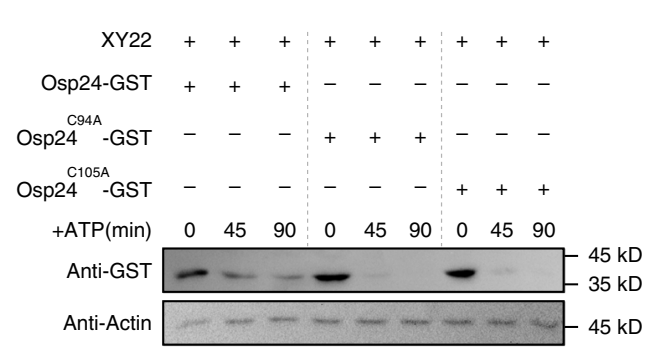

MCherry

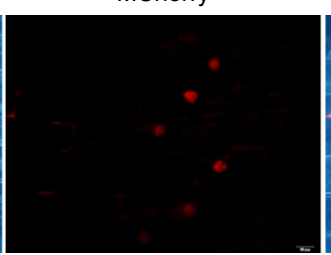

I
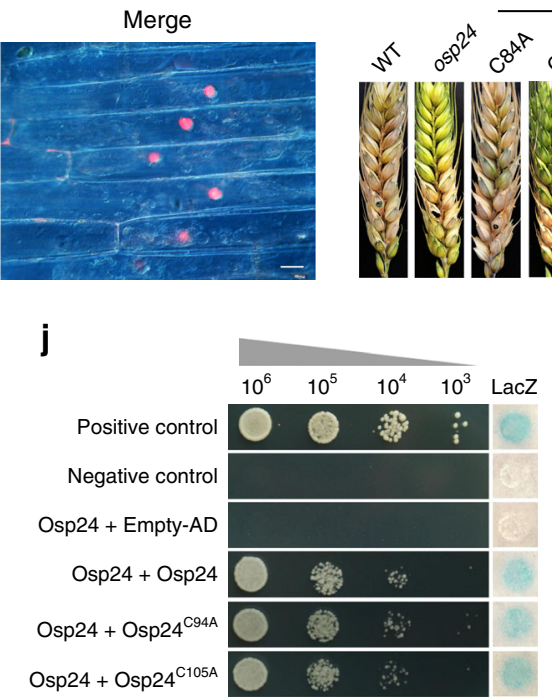

h

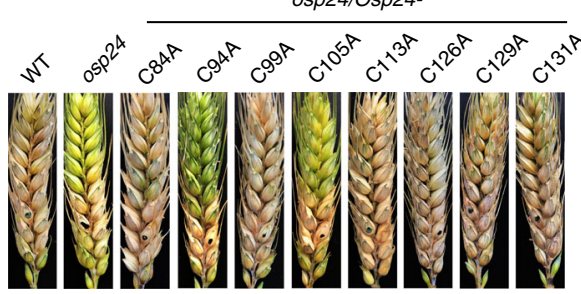

k

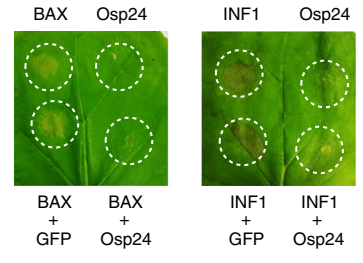

Fig. 2 Functions of Osp24 and its signal peptide and cysteine residues. a Infection cushions formed by the wild-type strain PH-1 (WT) and osp24 deletion mutant on wheat lemma at $2 \mathrm{dpi}$ were examined by SEM under $\times 800$ amplification. Scale bar, $10 \mu \mathrm{m}$. b. Thick sections of infected wheat heads were examined for invasive hyphae (red arrowheads) in the rachis tissues at $5 \mathrm{dpi}$. Scale bar, $20 \mu \mathrm{m}$. c Relative biomass of $F$. graminearum in infected wheat heads at 5 dpi was determined by qPCR. Mean and standard deviation were estimated with data from three $(n=3)$ independent biological replicates (marked as black dots on the bar). The asterisk * indicates significant differences $(P=0.05)$ based on Bootstrap analysis. $\mathbf{d}$ The positions of eight cysteine residues in Osp24 and the predicted intra-molecular disulfide bond between C94 and C105. SP, signal peptide. e The yeast suc2 mutant YTK12 and its transformants expressing the empty vector pSUC2 or vectors with the signal peptide from Osp24 and Avr1 (positive control) were assayed for growth on CMD-W or YPRAA plates and invertase activity in TTC medium. f Representative images of wheat heads infected with PH-1, osp24 mutant, and the osp24/OSP24 and osp24/OSP24 ${ }^{S P}$ transformants were photographed at 14 dpi. $\mathbf{g}$ Wheat coleoptiles were infected with $\mathrm{PH}-1$ expressing the Osp24: mCherry:NLS construct and examined for mCherry signals in plant cells. Scale bar, $20 \mu \mathrm{m}$. h Representative images of wheat heads infected with PH-1, osp24 mutant, and transformants of osp24 expressing OSP24 mutant alleles carrying the indicated C-to-A mutations were photographed at 14 dpi. i Western blots of mixtures of equal amounts of total proteins isolated from wheat heads of cultivar Xiaoyan 22 (XY22) and recombinant Osp24-GST,

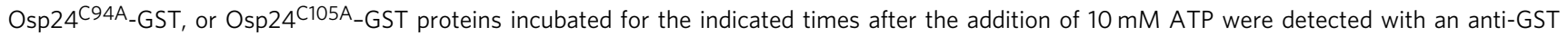
antibody. Detection with an anti-actin antibody was used as a loading control. $\mathbf{j}$ Different concentrations (cells/ml) of yeast transformants expressing the indicated bait and prey constructs were assayed for growth on SD-Trp-Leu-His plates and LacZ activity. $\mathbf{k}$ Transient expression of Osp24 suppressed programmed cell death triggered by BAX or INF1. At the indicated spots, N. benthamiana leaves were infiltrated with Agrobacterium cells expressing GFP/ BAX/INF1 alone or infiltrated with BAX/INF1-expressing cells at $18 \mathrm{~h}$ after infiltration with GFP or Osp24 first. Representative leaves were photographed 5 days after infiltration.

Osp24 (Fig. 2d) into the vector pSUC2 ${ }^{40}$ and transformed into the yeast suc2 mutant $^{41}$. The resulting SP-SUC2 transformants were able to grow on YPRAA agar and had secreted invertase activity (Fig. 2e), indicating that the SP of Osp24 is functional in yeast.

To test its function in F. graminearum, we then generated the OSP24 and OSP24 ${ }^{\triangle \mathrm{SP}}$ constructs ${ }^{42,43}$ and transformed them into the osp24 mutant. The resulting osp24/OSP24 transformants were normal in virulence. However, the osp $24 / O S P 24^{\Delta S P}$ transformants were similar to the osp 24 mutant in virulence on wheat heads
(Fig. 2f), indicating that the SP is essential for the complementation of osp24 mutant by ectopic expression of OSP24. Therefore, the secretion of Osp24 is important for its function during plant infection.

Osp24 is a cytoplasmic effector that is translocated into plant cells. To determine whether Osp24 is delivered into plant cells, we generated the $\mathrm{P}_{\mathrm{OSP} 24}$-OSP24-mCherry-NLS construct with the NLS from SV40 T-Antigen and transformed it into PH-1. In wheat seedlings infected with transformants expressing the 
Osp24-mCherry-NLS construct, mCherry signals were observed in the nucleus in coleoptile cells at the inoculation sites (Fig. 2g), suggesting that Osp24 is a cytoplasmic effector that is translocated into plant cells.

The C94 and C105 cysteine residues are important for the function of Osp24. Similar to many other fungal effectors, Osp24 is a cysteine-rich protein with eight cysteine (C) residues (Fig. 2d). To determine their roles in Osp24 function, constructs of OSP24 with individual Cs changed to alanine (A) were generated and transformed into the osp 24 mutant. Whereas mutations of six other cysteine residues had no effect on virulence, the osp24/OSP24 $494 \mathrm{~A}$ and osp24/OSP24 $105 \mathrm{~A}$ transformants, similar to the osp 24 mutant, were defective in wheat head infection (Fig. 2h), indicating that $\mathrm{C} 94$ and $\mathrm{C} 105$ are essential for the function of Osp24 proteins during pathogenesis.

When analyzed for the formation of intra-molecular disulfide bonds with the EDBCP tool ${ }^{44}$, the disulfide bridge between C94 and C105 was predicted with the highest probability $($ Probability $=0.89774)$. To characterize their functions, we expressed and purified Osp24-, Osp24 ${ }^{\mathrm{C} 94 \mathrm{~A}_{-} \text {, and Osp24C105A }}$ GST fusion proteins. Equal amounts of these recombinant proteins were co-incubated with total proteins isolated from wheat heads inoculated with $\mathrm{PH}-1$ sampled at $3 \mathrm{dpi}$ and assayed for their degradation by western blot analyses. In comparison with Osp24-GST proteins that were degraded gradually over time, the degradation of Osp24 ${ }^{\mathrm{C} 94 \mathrm{~A}}$ and Osp24 ${ }^{\mathrm{C} 105 \mathrm{~A}}$ GST fusion proteins occurred much more rapidly (Fig. 2i), indicating that Osp24 proteins may be less stable when $\mathrm{C} 94$ or $\mathrm{C} 105$ is changed to A. Therefore, the intra-molecular disulfide bond between C94 and C105 may be important for the folding and stability of Osp24 proteins.

When tested by yeast two-hybrid assays, the Osp24 protein was found to interact with itself (Fig. 2j), suggesting that Osp24 may form homodimers. To determine their roles in the formation of homodimers, we also generated the bait constructs of Osp24 carrying the C94A or C105A mutation. Similar to the Osp24-Osp24 interaction, Osp24 interacted with both Osp24 $4^{\mathrm{C} 94 \mathrm{~A}}$ and Osp24 ${ }^{\mathrm{C} 105 \mathrm{~A}}$ in yeast two-hybrid assays (Fig. 2j), indicating that the C-to-A mutation at $\mathrm{C} 94$ or $\mathrm{C} 105$ had no obvious effect on its intermolecular interaction. Therefore, C94 and C105 are likely only important for the formation of intramolecular disulfide bonds.

Osp24 suppresses cell death and the expression of metabolismrelated genes. To characterize its role as an effector to suppress host immunity, we first assayed the effect of Osp24 on programmed cell death (PCD) in Nicotiana benthamiana induced by BAX, a mouse proapoptotic protein ${ }^{45}$. The $\mathrm{P}_{\mathrm{CaMV}} 35 \mathrm{~s}^{-\mathrm{O}} \mathrm{Osp} 24^{\Delta \mathrm{SP}}$ construct was generated and transformed into Agrobacterium tumefaciens. In $N$. benthamiana leaves infiltrated with Agrobacterium expressing Osp24 ${ }^{\Delta \mathrm{SP}}$ alone, no cell death was observed. Under the same conditions, PCD was observed on leaves infiltrated with cells expressing BAX. However, on leaves infiltrated with $A$. tumefaciens expressing Osp2 $4^{\triangle \mathrm{SP}} 18 \mathrm{~h}$ before infiltration with cells expressing BAX, cell death was not observed when Osp24 $4^{\triangle \mathrm{SP}}$ and BAX proteins were co-expressed (Fig. 2k, Supplementary Fig. 6), indicating suppression of BAX-induced cell death by Osp24. We also tested the effects of Osp24 on PCD triggered by INF1, a pathogen-associated molecular pattern (PAMP) from Phytophthora infestans ${ }^{46}$. In infiltration assays with $N$. benthamiana leaves, INF1-induced cell death also was suppressed by Osp24 (Fig. 2k).

To identify genes suppressed or induced by Osp24 during plant infection, we conducted RNA-seq analysis with infected wheat heads that were collected at 3 dpi. A total of 478 differentially expressed genes (DEGs) with at least two-fold changes were identified in wheat infected with the wild type and osp24 mutant. Among them, 296 DEGs were up-regulated in wheat inoculated with the osp 24 mutant, suggesting that their expression may be suppressed by Osp24 during F. graminearum infection. FunCat-enrichment analysis showed that up-regulated DEGs were significantly enriched for genes functionally related to metabolism, information pathway, and perception and response to stimuli (Supplementary Fig. 7). Gene Ontology (GO)-enrichment analysis revealed that many GO categories associated with metabolism were significantly enriched, including oxidation-reduction, glycolytic, glycine catabolic, malate metabolic, peroxisome fission, chlorophyll biosynthetic, fructose 2,6-bisphosphate metabolic, fructose metabolic, L-serine metabolic, photosynthesis, and ATP synthesis coupled proton transport processes (Supplementary Data 1). Interestingly, 17 $(\sim 7 \%)$ of the up-regulated DEGs in osp24-infected wheat heads encode putative NBS-LRR proteins that are known to be involved in plant immunity against microbial pathogens ${ }^{47}$ Putative NBS-LRR genes were absent in the down-regulated DEGs. It is possible that some of these NBS-LRR genes with upregulated expression in osp24-infected wheat heads may contribute to defense responses against $F$. graminearum infection.

In comparison with the wild type, only 54 and 83 fungal genes were up-regulated and down-regulated, respectively, in the osp24 mutant during wheat infection. Because none of them are related to known virulence factors or putative effector genes (Supplementary Data 2), their altered expression levels may not contribute significantly to the defect of the osp24 mutant in virulence.

Osp24 interacts with wheat TaSnRK1a. To further characterize its function during plant infection, we generated an Osp24 bait construct and screened a yeast two-hybrid library constructed with RNA isolated from infected wheat heads collected at $3 \mathrm{dpi}$. After screening over five coverage of this library, 16 putative Osp24-interacting clones (OICs) were identified (Supplementary Table 4). Sequencing analysis showed that five clones were the SNF1-related protein kinase gene TaSnRK $1 \alpha^{28}$ that is highly similar to Arabidopsis SnRK1. In Arabidopsis, SnRK1 is a central integrator of stress and energy signaling that regulates plant metabolism, growth, and immunity 48,49 .

The wheat genome has three TaSnRK1a homoelogues (A, B, and D) that share the same AA sequence and have only 12-14 nucleotide differences in the coding regions ${ }^{50}$. Because all the five TnSnRK1a clones identified in the original yeast two-hybrid library screen were TaSnRK1 $\alpha-A$, we used TnSnRK1 $\alpha-A$ for all the experiments related to TaSnRK1 $\alpha$ unless otherwise stated. We then generated the full-length TaSnRK1a prey construct and confirmed its interaction with Osp24 in yeast two-hybrid assays (Fig. 3a). To verify their interaction in plant cells, the Osp24-nYFP and TaSnRK1a-cYFP fusion constructs were generated and coexpressed in N. benthamiana leaves by Agrobacterium infiltration. In epidermal cells of infiltrated tobacco leaves, YFP signals were observed in the nucleus but not in the cytoplasm (Fig. 3b), indicating the expression of Osp24-nYFP and TaSnRK1 $\alpha$-cYFP and their interaction in the nucleus of plant cells. We further verified the interaction between Osp24 and TaSnRK1a by in vitro pull-down assays. Recombinant Osp24-GST and TaSnRK1a-HIS proteins were purified from Escherichia coli and co-incubated with glutathione resins. In western blots of proteins eluted from glutathione resins, the TaSnRK1a-HIS band was detected (Fig. 3c), indicating its interaction with Osp24-GST in vitro. 
a

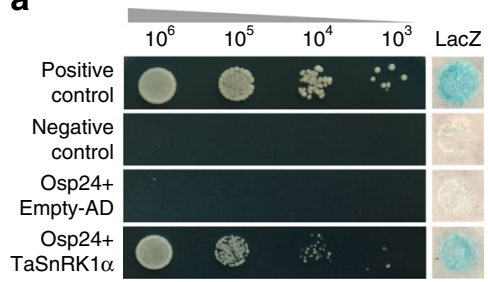

C

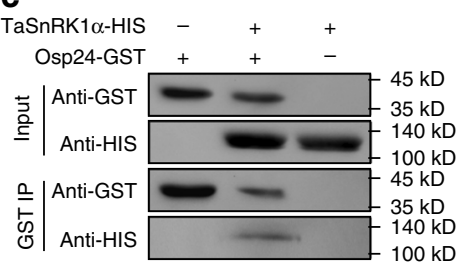

b

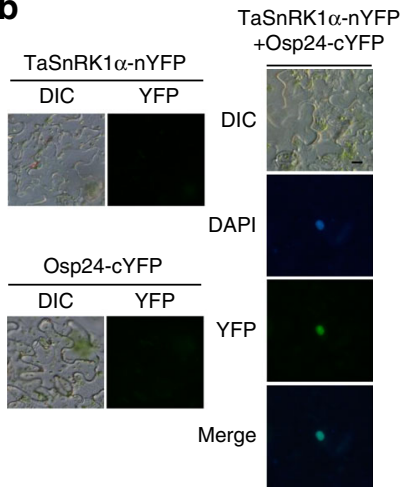

d

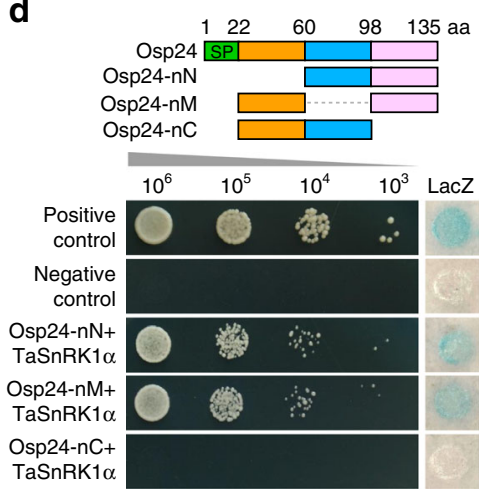

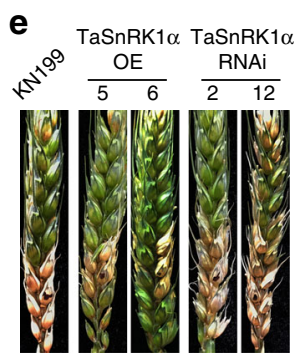

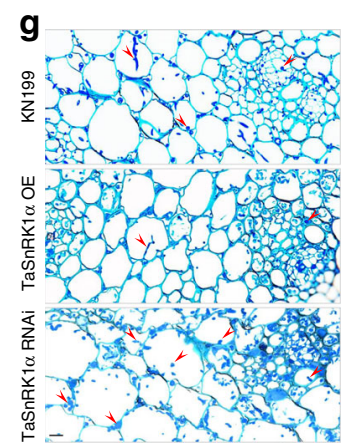

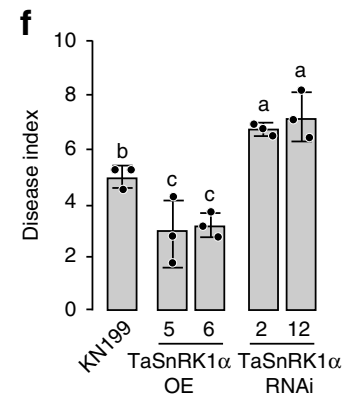

h

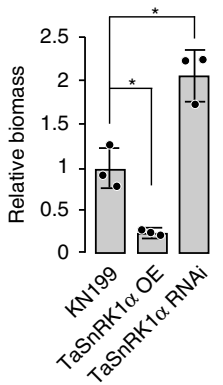

Fig. 3 Interaction of Osp24 with TaSnRK1 $\alpha$ and function of TaSnRK1 $\alpha$ in wheat resistance against $\boldsymbol{F}$. graminearum. a Yeast two-hybrid assays to detect the interaction of Osp24 (Bait) with TaSnRK1 $\alpha$ (Prey). Different concentrations of the labeled yeast transformants were assayed for growth on SD-Trp-LeuHis plates and LacZ activity. $\mathbf{b}$ BiFC assays for the interaction of Osp24 with TaSnRK1 $\alpha$. Leaves of $N$. benthamiana were agroinfiltrated with a mixture of $A$. tumefaciens strains expressing the Osp24-nYFP and TaSnRK1 $\alpha$-cYFP constructs. YFP signals were observed at 2 days post-agroinfiltration. Infiltration with Agrobacterium expressing the TaSnRK1 $\alpha$ or Osp24 construct alone was used as the negative control. No YFP signals was observed in these negative controls. Scale bar, $20 \mu \mathrm{m}$. c Verification of the Osp24-TaSnRK1 $\alpha$ interaction by GST pull down assays. Western blots of the marked protein mixtures (Input) or proteins co-purified with TaSnRK1 $\alpha$-HIS from these mixtures (GST IP) were detected with the anti-HIS and anti-GST antibodies. d Yeast transformants expressing the TaSnRK1 $\alpha$ (prey) and marked full-length or truncated Osp24 (bait) were assayed for growth on SD-Trp-Leu-His plates and LacZ activities. SP signal peptide. e Representative wheat heads of cultivar KN199 and its transgenic lines expressing the TaSnRK1 $\alpha$ overexpression (TaSnRK1 $\alpha$ OE 5 and 6) or silencing (TaSnRK1 $\alpha$ RNAi 2 and 12) construct were drop-inoculated with PH-1 and photographed at 7 dpi. f Mean and standard deviation (SD) of the disease index of PH-1 on the labeled wheat lines were estimated from three independent experiments $(n=3)$ with at least 10 infected wheat heads in each experiment. Different letters indicate significant differences based on ANOVA analysis followed by Duncan's multiple range test $(P=0.05)$. $\mathbf{g}$ Thick sections of the rachises of wheat heads of KN199 and its TaSnRK1 $\alpha$ OE or TaSnRK1 $\alpha$ RNAi transgenic line inoculated with PH-1 were examined for invasive hyphae (red arrowheads) at $5 \mathrm{dpi}$. Scale bar, $50 \mu \mathrm{m}$. h Relative biomass of $F$. graminearum was determined by qPCR in infected wheat heads of KN199 and labeled transgenic lines sampled at 5 dpi. Mean and standard deviation were estimated with data from three $(n=3)$ independent biological replicates (indicated with black dots on the bar). *Indicates significant differences $(P=0.05)$ based on Bootstrap analysis.

To determine the region required for its association with TaSnRK1a, three different fragments of Osp24 (Fig. 3d) were amplified and tested in yeast two-hybrid assays. Deletion of the C-terminal region (aa 99-135) of Osp24 eliminated its interaction with TaSnRK1a (Fig. 3d). However, deletion of the N-terminal (aa 1-60) or middle (aa 61-98) region had no obvious effect on the Osp24-TaSnRK1a interaction (Fig. 3d). Therefore, the Cterminal region of Osp24 is involved in its interaction with TaSnRK1a. In Agrobacterium infiltration assays, expression of Ops24 with a truncated C-terminal region failed to suppress BAX-induced or INF1-induced cell death in $N$. benthamiana (Supplementary Fig. 8). These results indicate that the C-terminal region of Osp24 is related to its function in PCD suppression, likely by interacting with SnRK1.

TaSnRK1a contributes to resistance against $F$. graminearum. To determine its function in resistance against $F$. graminearum, we first generated a TaSnRK1 $\alpha$ RNAi construct and transformed it into wheat cultivar KN19951, which is a cultivar amenable to efficient transformation but more tolerant to FHB than cultivar Xiaoyan 22. In transgenic lines, the expression level of TaSnRK1a was reduced approximately two-fold based on qRT-PCR assays (Supplementary Fig. 9a). In wheat head infection assays, transgenic lines with reduced TaSnRK1a expression displayed more severe FHB symptoms than cultivar KN199 when inoculated with the wild-type strain PH-1 (Fig. 3e). The disease index of PH-1 was increased in lines expressing the TaSnRK1a RNAi construct in comparison with KN199 (Fig. 3f).

We also generated transgenic lines with the TaSnRK1a gene under the control of the CaMV $35 \mathrm{~S}$ promoter ${ }^{52}$. Increased expression of TaSnRK1a (over 3-fold) was detected in five transgenic lines (Supplementary Fig. 9b). In wheat head infection assays, transgenic plants overexpressing TaSnRK1a were increased in resistance to F. graminearum (Fig. 3e). The disease index of PH-1 on TaSnRK1a overexpressing plants was decreased $\sim 40 \%$ compared to that on KN199 (Fig. 3f).

Overexpression of TaSnRK1a also significantly reduced infectious growth and only limited invasive hyphae were observed in the rachis tissues of transgenic wheat plants overexpressing TaSnRK1a (Fig. 3g). When assayed by qPCR, less fungal biomass was detected in infected wheat heads in transgenic lines overexpressing TaSnRK1a than in KN199 (Fig. 3h). In contrast, more abundant infectious hyphae were observed in the rachis tissues (Fig. $3 \mathrm{~g}$ ), and more fungal biomass was detected by qPCR (Fig. 3h) in wheat heads of transgenic plants expressing the TaSnRK1a RNAi construct than in the control KN199 plants. Nevertheless, DON production in the diseased spikelets inoculated with PH-1 was similar between KN199 and TaSnRK1a 
RNAi plants (Supplementary Fig. 10). These results indicate that overexpression of TaSnRK1a in wheat plants increased the resistance against $F$. graminearum and silencing of TaSnRK1a had the opposite effect.

Osp24 accelerates TaSnRK1a degradation during infection. To assay the effect of Osp24 on TaSnRK1a proteins in a cell-free degradation assay, equal amounts of TaSnRK1 $\alpha$-HIS recombinant proteins were mixed and co-incubated with total proteins isolated from wheat heads of cultivar Xiaoyan 22 inoculated with the wild-type strain $\mathrm{PH}-1$ or osp 24 mutant (sampled at $3 \mathrm{dpi}$ ). In the presence of ATP, TaSnRK1a was degraded over time (45-90 min) in both co-incubation mixtures (Fig. 4a). However, the rate of TaSnRK1 degradation was reduced in co-incubation mixtures with proteins from osp24-infected wheat heads in comparison with those with proteins from PH-1-infected samples (Fig. 4a). These results suggest that Osp24 may accelerate TaSnRK1a degradation during infection. Under the same conditions, TaSnRK1a degradation was not observed in the co-incubation mixture of TaSnRK1a-HIS and Osp24-GST without proteins from infected wheat heads (Fig. 4b). However, the degradation of TaSnRK1a-HIS was observed in its co-incubation mixture with Osp24-GST and protein extracts from non-inoculated wheat heads (Fig. 4c), indicating that Osp24-stimulated degradation of TaSnRK1a is dependent on some components of wheat head protein extracts.

We also assayed the transcription level of TaSnRK1a during $F$. graminearum infection. TaSnRK1a transcription was not upregulated in infected wheat heads in comparison with noninoculated samples (Supplementary Fig. 11a). Furthermore, wheat plants inoculated with $\mathrm{PH}-1$ or the osp 24 mutant had no obvious difference in the abundance of TaSnRK1 $\alpha$ transcripts (Supplementary Fig. 11b). These results indicate that Osp24 does not affect the transcription of TaSnRK1a but may stimulate its degradation in infected wheat heads.

Osp24 facilitates the interaction of TaSnRK1a with the ubiquitin-26S proteasome system. In Arabidopsis, SnRK1 interacts with both the SCF ubiquitin ligase complex and $26 \mathrm{~S}$ proteasome ${ }^{53}$. Osp24 may promote the degradation of TaSnRK1a through the ubiquitin-26S proteasome pathway ${ }^{54}$ during fungal infection. To test this hypothesis, we first assayed the effect of proteasomal inhibitor MG132 on TaSnRK1a degradation. Even in the presence of Osp24, the degradation of TaSnRK1a in comixtures with proteins isolated from infected wheat heads was suppressed by MG132 (Fig. 4d), indicating the involvement of the $26 \mathrm{~S}$ proteasome.

We then investigated the effects of Osp24 on the interaction between TaSnRK1a and the ubiquitin-26S proteasome by in vitro pull-down assays. TaSnRK1a-HIS proteins were co-incubated with total proteins isolated from wheat heads in the presence or absence of Osp24-GST for 30 min before mixing with anti-HIS beads. Proteins bound to anti-HIS beads were then eluted and assayed for the presence of SCF ubiquitin ligase complex and 26S proteasome with the anti-RBX1 and anti-20S proteasome antibodies $^{37}$, respectively. With or without the addition of Osp24-GST, both SCF ubiquitin ligase complex and 26S proteasome were detected in proteins co-immunoprecipitated with TaSnRK1a-HIS (Fig. 4e). However, the presence of Osp24 increased the amount of these proteins co-purified with TaSnRK1a-HIS (Fig. 4e). These results indicate that Osp24 may accelerate TaSnRK1 $\alpha$ degradation by enhancing its association with the ubiquitin-26S proteasome system.
The wheat orphan protein TaFROG competes with Osp24 in binding with TaSnRK1 $\alpha$. TaSnRK1 $\alpha$ is known to interact with a wheat orphan protein TaFROG, which is induced by $\mathrm{DON}^{28}$. In yeast two-hybrid assays, the C-terminal region of TaSnRK1a (267-499 aa) was found to interact with both Osp24 and TaFROG (Fig. 4f). In contrast, the N-terminal region of TaSnRK1a (1-266 aa) was dispensable for its interaction with Osp24 or TaFROG. The direct interaction between Osp24 and TaFROG was not detected in yeast two-hybrid assays (Supplementary Fig. 12).

Because both Osp24 and TaFROG interacted with the same region of TaSnRKla, it is possible that they may compete with each other for interacting with TaSnRK1a. To test this hypothesis, TaSnRK1a-HIS, Osp24-GST, and TaFROG-S-tag fusion proteins were purified and used in in vitro pull-down assays. When equal amounts of TaSnRK1 $\alpha$-HIS and Osp24-GST proteins were co-incubated and mixed with anti-HIS beads, abundant TaSnRK1a proteins were detected in proteins copurified with Osp24-GST. With the addition of increasing concentrations of TaFROG proteins to the TaSnRK1a-Osp24 protein mixtures, the amount of TaSnRK1a proteins co-purified with Osp24-GST was gradually reduced in a concentrationdependent manner (Fig. 4g), indicating the competition between TaFROG and Osp24 in binding with TaSnRK1a.

Overexpressing TaFROG enhances FHB resistance by stabilizing TaSnRK1a. To determine the role of TaFROG in regulating TaSnRK1a stability during F. graminearum infection, we generated transgenic wheat plants in which the TaFROG gene was overexpressed with the CaMV $35 \mathrm{~S}$ promoter. When assayed by qRT-PCR, the expression level of TaFROG was increased over 100-fold in five transgenic lines (Supplementary Fig. 13). In wheat head infection assays, fewer spikelets developed FHB symptoms in the TaFROG overexpression transgenic lines than the control KN199 plants (Fig. 4h, i), indicating an increase in resistance against $F$. graminearum by TaFROG overexpression, which is consistent with an earlier report ${ }^{28}$. We then conducted cell-free degradation assays with equal amounts of TaSnRK1a-HIS recombinant proteins co-incubated with total proteins isolated from wheats heads of control KN199 and TaFROG overexpression plants infected with PH-1. Although TaSnRK1a degradation was observed in all the reaction mixtures, the degradation rate was significantly reduced in co-incubation mixtures with proteins isolated from transgenic lines overexpressing TaFROG in comparison with those from KN199 plants (Fig. 4j), confirming that overexpression of TaFROG increases the stability of TaSnRK1a. In the same degradation assays, the rate of TaSnRK1a degradation was similar in coincubation mixtures with proteins isolated from wheat heads infected with PH-1 or the osp24 mutant (Supplementary Fig. 14). Therefore, overexpression of TaFROG may enable its effective protection of TaSnRK1a against Osp24 binding and degradation.

TaFROG may compete with Osp24 in binding with TaSnRK1a and reduce its degradation via the ubiquitin-26S proteasome system. To test this hypothesis, TaSnRK1 $\alpha$-HIS proteins were mixed with total proteins isolated from wheat heads infected with $\mathrm{PH}-1$ and anti-HIS beads. Western blots of proteins eluted from anti-HIS beads were then detected with the anti-RBX1 and anti20s proteasome antibodies. The density of both SCF ubiquitin ligase complex and $26 \mathrm{~S}$ proteasome bands was weaker in samples with proteins isolated from transgenic plants overexpressing TaFROG than those with proteins from KN199 (Fig. 4k). These results indicate that overexpression of TaFROG may reduce the interaction of TaSnRK1a with the ubiquitin-26S proteasome 
a

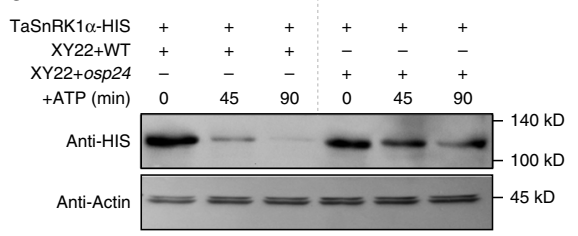

d

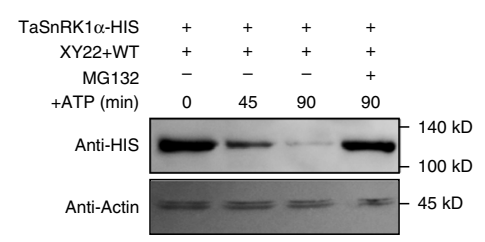

g

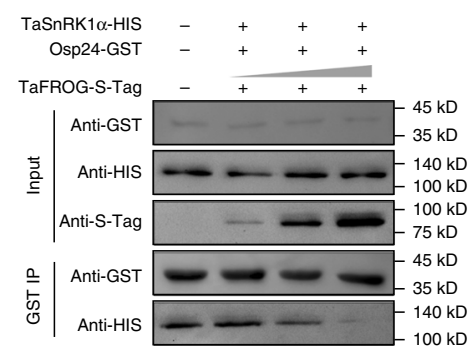

h

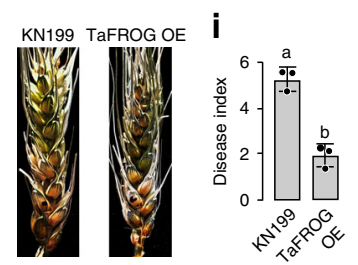

b

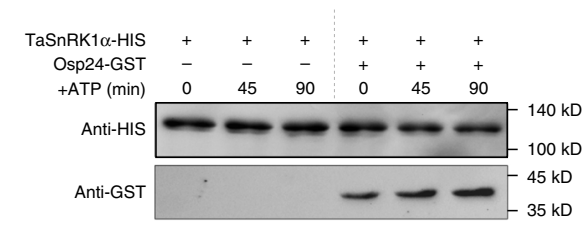

e

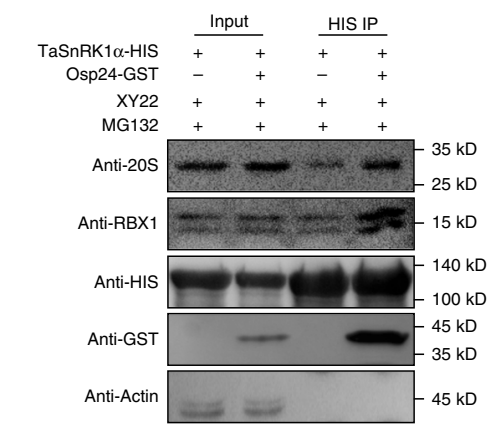

C

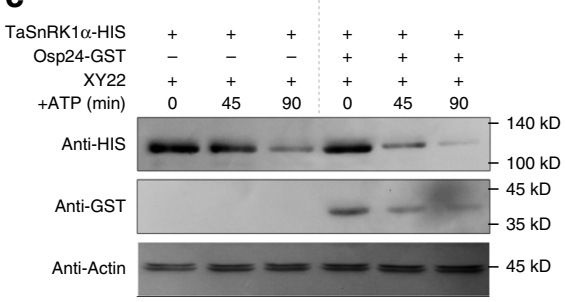

f

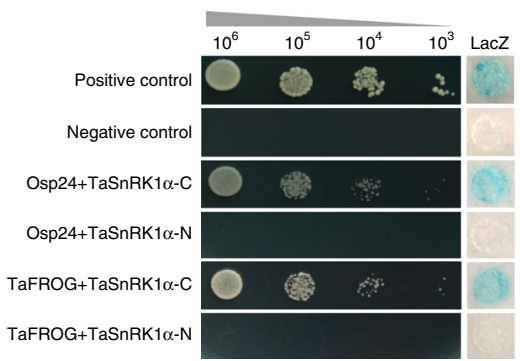

k

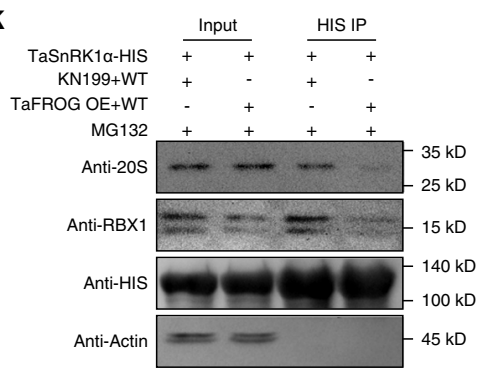

Fig. 4 Roles of Osp24 and TaFROG in modulating TaSnRK1 $\alpha$ stability. a Western blots of the mixtures of equal amount of TaSnRK1 $\alpha-H I S$ and total proteins isolated from wheat heads of cultivar Xiaoyan22 inoculated with the wild type PH-1 (XY22 + WT) or osp24 mutant (XY22 + osp24) incubated for the indicated times after the addition of $10 \mathrm{mM} \mathrm{ATP}$ were detected with the anti-HIS or anti-actin (loading control) antibody. $\mathbf{b}$ Western blots of the mixtures of equal amount of TaSnRK1 $\alpha$-HIS and Osp24-GST incubated for the indicated times were detected with an anti-HIS or anti-GST antibody. Degradation of TaSnRK1 $\alpha$-HIS by Osp24 was not observed. c Recombinant TaSnRK1 $\alpha$-HIS and Osp24-GST proteins were incubated with equal amounts of total proteins isolated from wheat head (XY22) in the presence of $10 \mathrm{mM}$ ATP and used for western blot analyses with the indicated antibodies.

d Degradation of TaSnRK1 $\alpha$ by total proteins isolated from wheat head inoculated with PH-1 (XY22 + PH-1) was inhibited by proteasome inhibitor MG132. e Western blots of the indicated protein mixtures (Input) or proteins co-purified with TaSnRK1 $\alpha$-HIS from these protein mixtures (HIS IP) were detected with the anti-20S, anti-RBX1, anti-HIS, and anti-GST antibodies. $f$ Yeast transformants expressing the indicated Osp24 or TaFROG bait construct and prey constructs of the N-terminal (1-266 aa) or C-terminal (267-499 aa) region of TaSnRK1 $\alpha$ (TaSnRK1 $\alpha-\mathrm{N}$ and TaSnRK1 $\alpha-\mathrm{C}$ ) were assayed for growth on SDTrp-Leu-His plates and LacZ activity. $\mathbf{g}$ Western blots of the indicated protein mixtures (Input) or proteins co-purified with Osp24-GST from these mixtures (GST IP) were detected with the anti-GST, anti-HIS, or anti-S-tag antibody. The amount of TaSnRK1 $\alpha$-HIS proteins co-immunoprecipitated with Osp24-GST was reduced by the addition of increasing concentrations of TaFROG-S-tag proteins. $\mathbf{h}$ Representative wheat heads of cultivar KN199 and its TaFROG overexpressing transgenic line (TaFROG OE) infected with PH-1 were photographed at 8 dpi. i Mean and standard deviation (SD) of the disease index of $\mathrm{PH}-1$ on the labeled wheat lines were estimated from three independent experiments $(n=3)$ with at least 10 infected wheat heads in each replicate. Different letters indicate significant differences based on ANOVA analysis followed by Duncan's multiple range test $(P=0.05)$. $\mathbf{j}$ Cell-free degradation assays with recombinant TaSnRK1 $\alpha$-HIS incubated with equal amounts of total proteins isolated from wheat heads of KN199 and TaFROG OE transgenic lines infected by PH-1 for the indicated time after addition of 10 mM ATP. $\mathbf{k}$ Western blots of the indicated protein mixtures (Input) or proteins co-purified with TaSnRK1 $\alpha$-HIS from these protein mixtures (HIS IP) were detected with the anti-20S, anti-RBX1, and anti-HIS antibodies. For $\mathbf{a}$, c, d, e, j, and $\mathbf{k}$, detection with an anti-actin antibody was used as the control.

system in these transgenic plants, possibly by competing with Osp24.

\section{Discussion}

Like many other plant pathogenic fungi, F. graminearum may use secreted proteins or effectors to suppress plant defense responses and its genome contains hundreds of orphan genes that are specifically expressed or highly up-regulated during plant infection. In this study, a total of 50 secretory proteins unique to $F$. graminearum were identified and functionally characterized. Among them, OSP24, OSP25, and OSP44, were found to be important for virulence. They all have typical features of fungal effectors $^{4,5}$ and are in the fast-evolving telomeric regions ${ }^{55}$. The location of these three putative effector genes in the telomeric regions may allow the rapid gain and loss or translocation to supernumerary chromosomes as effector reservoirs ${ }^{39,56}$ although supernumerary chromosomes have not been reported in the few sequenced $F$. graminearum strains.

OSP24 was selected for further characterization because the osp24 deletion mutant had the most significant reduction in virulence. Although dispensable for vegetative growth, reproduction, and initial penetration, OSP24 is important for infectious 
growth in the rachis tissues in infected wheat heads. Osp24 lacks any conserved domain but is a cysteine-rich protein. Two of the eight cysteine residues, C94 and C105, were found to be important for the function of Osp24 in plant infection. In Sclerotinia sclerotiorum, two cysteine residues of effector SsSSVP1 are essential for the formation of a homo-dimer and its interaction with the host target ${ }^{57}$. Although Osp24 may form homo dimers, C94 and C105 were dispensable for the Osp24-Osp24 interaction in yeast two-hybrid assays. In fact, these two cysteine residues were predicted to form an intra-molecular disulfide bond. In Stagonospora nodorum, cysteine residues of effector SnTox1 may form multiple disulfide bonds to resist degradation ${ }^{58}$. In F. graminearum, the formation of a disulfide bond between C94 and C105 may be important for the proper folding and stability of Osp24 in infected wheat tissues.

Osp24 is a cytoplasmic effector that is translocated into plant cells during infection. In $M$. oryzae, a number of cytoplasmic effectors are accumulated in the BIC before being delivered into plant cells ${ }^{34}$. It is possible that Osp24 is translocated into wheat cells through BIC-like structures that may be formed by F. graminearum. Osp24 strongly interacted with TaSnRK1a in yeast two-hybrid assays and their interactions were confirmed by BiFC and in vitro pull-down assays. The highly conserved SnRK1 kinases function as metabolic regulators of energy homeostasis and are important for development and stress responses in plants $^{48,59}$. SnRK1 kinases also are involved in regulating plant immunity and known to be targeted by viral and bacterial effector proteins ${ }^{60-63}$. Although SnRK1 is likely a conserved target for fungal pathogens, interactions between fungal secretory proteins and plant SnRK1 kinases have not been reported. In rice, the expression of OsSnRK1a was reported to be associated with disease resistance against $M$. oryzae, Cochliobolus miyabeanus, and Rhizoctonia solani ${ }^{64,65}$ but its exact role in disease resistance has not yet been characterized. In wheat, the kinase activity of TaSnRK1 $\alpha$ was increased in the presence of DON produced by $F$. graminearum $^{50}$. In this study, we showed that overexpression of TaSnRK1 $\alpha$ in wheat increased resistance against $F$. graminearum but transgenic plants expressing the SnRK1 silencing construct were more susceptible. It is likely that TaSnRK1a plays an important role in regulating resistance responses to $F$. graminearum and is a target of effector protein Osp24 secreted by the pathogen during infection.

The interaction of Osp24 with TaSnRK1a likely results in its degradation in infected wheat heads because the degradation of TaSnRK1 $\alpha$ was accelerated in the presence of Osp24 in cell-free degradation assays. In Arabidopsis, the degradation of SnRK1 kinases is commonly associated with sumoylation and ubiquitylation $^{66}$. The association of SnRK1 kinases with SCF ubiquitin ligases complex and components of the $26 \mathrm{~S}$ proteasome also have been well documented ${ }^{53}$. In this study, we showed that treatments with MG132, an inhibitor of the $26 \mathrm{~S}$ proteasome ${ }^{67}$, reduced Osp24-mediated TaSnRK1a degradation. Furthermore, we showed that Osp24 increased the association of TaSnRK1a with the SCF ubiquitin ligase complex and 26S proteasome, indicating that the stimulation of TaSnRK1a degradation by Osp24 likely occurs through the ubiquitination-26S proteasomal pathway ${ }^{67}$. Fungal effectors are known to target the ubiquitinproteasome pathway in other pathosystems. Whereas effectors Pit2 of $U$. maydis and Avr2 of Cladosporium fulvum inhibit host proteases that are required for basal defense ${ }^{68,69}$, AvrPiz-t of $M$. oryzae suppresses the RING E3 ubiquitin ligase APIP6 in plant cells $^{70}$. Our results suggest that Osp24 is secreted by F. graminearum as an effector interacting with TaSnRK1 $\alpha$ to stimulate its ubiquitination and proteasomal degradation, which in turn negatively impacts resistance responses to fungal infection in wheat plants.
Transient expression of Osp24 but not Osp24 truncated of its C-terminal region suppressed BAX-induced or INF1-induced cell death in $N$. benthamiana. Because the C-terminal region of Osp24 mediates its interaction with TaSnRK1 $\alpha$, it is possible that Osp24 suppresses PCD by targeting $N$. benthamiana SnRK1. The role of SnRK1 in PCD suppression was also reported in Pepper. Silencing of the pepper SnRK1 transcript resulted in a significant reduction of hypersensitive response (HR) that is elicited by protein AvrBs1 from Xanthomonas campestris pv. vesicatoria $(X c v)^{62}$. The YopJ effector homolog AvrBsT from Xcv targeted SnRK1 to suppress AvrBs1-induced plant immunity ${ }^{62}$.

In F. graminearum, DON is not essential for the initial infection but plays a critical role in the spreading of infectious growth via the rachis in infected wheat heads. However, DON is not important for infecting Arabidopsis floral tissues ${ }^{71}$, likely because infectious growth can spread by hyphae grown on the surface of floral tissues. Interestingly, the expression of TaFROG that encodes a Pooideae-specific orphan protein was induced by DON treatment. TaFROG interacts with TaSnRK1a, and overexpression of TaFROG increased resistance against F. graminearum $^{28}$. In this study, we showed that Osp24 and TaFROG interacted with the same region of TaSnRK1a. Furthermore, we showed that their binding with TaSnRK1 $\alpha$ was competitive and the binding of TaFROG with TaSnRK1a increased its stability (Fig. 5). It is likely that TaFROG functions in defense against $F$. graminearum by protecting TaSnRK1 $\alpha$ against Osp24-mediated degradation. A recent report showed that TaFROG also interacts with TaNACL-D1, a Poaceae-divergent NAC transcription factor with its NAC C-terminal region specific to the Triticeae, to enhance FHB resistance independent of its interaction with TaSnRK1 $1 \alpha^{29}$. It is possible that Osp24 also interacts with TaNACL-D1 to suppress defense against FHB. Osp24 and TaFROG are orphan proteins in the pathogens and hosts, respectively, and each may be subjected to co-evolution during fungal-plant interactions. To our knowledge, the active adoption of competing orphan proteins in both fungal pathogen and plant hosts has not been reported in other pathosystems. Expressing engineered TaFROG alleles with stronger interactions with TaSnRK1a or using the host-induced gene silencing (HIGS) approach to silence OSP24 may improve resistance against F. graminearum without yield penalties because they encode orphan proteins specifically expressed during infection.

\section{Methods}

Identification of OSPs in F. graminearum. The genome and predicted protein sequences of F. graminearum, F. verticillioides, and F. oxysporum, were downloaded from the Broad Institute website (ftp://ftp.broadinstitute.org/pub/annotation/fungi/ fusarium/). To identify unique genes, protein sequences of $F$. graminearum were first used as the queries to search against the predicted proteomes of $F$. verticillioides and $F$. oxysporum by BLASTp. Sequences of the F. graminearum proteins also were used to search against the genome sequences of $F$. verticillioides and F. oxysporum by tBLASTn for possible genes that might not be predicted by automated annotation. (This study was initiated in 2010 when only the genome sequences of $F$. verticillioides and $F$. oxysporum were publicly available as the most closely related species of $F$. graminearum.) All protein sequences of $F$. graminearum without homologs in these searches ( $E$ value cutoff of $1 \mathrm{e}-5$ ) were extracted and then used as queries to search against NCBI number database (excluding F. graminearum sequences) by BLASTp. The proteins without detectable homologs in these searches ( $E$ value cut off of $1 \mathrm{e}-5)$ were considered as orphan proteins of $F$. graminearum. Some of them are not unique to $F$. graminearum because they have homologs in the genome sequences of other Fusarium species that late became available in the public domain. Therefore, these sequences were described as orphan genes in F. graminearum and its close-related species. These predicted orphan proteins were further analyzed with SignalP 3.0 (http://www.cbs.dtu.dk/ services/SignalP-3.0/) to identify secretory proteins.

Culture conditions and fungal transformation. The wild-type $F$. graminearum strain $\mathrm{PH}-1^{33}$ and deletion mutants of OSP genes were routinely cultured on potato dextrose agar (PDA) plates at $25^{\circ} \mathrm{C}$. PDA cultures grown at $25^{\circ} \mathrm{C}$ were used for measuring growth rate or colony morphology. Conidiation was assayed with 


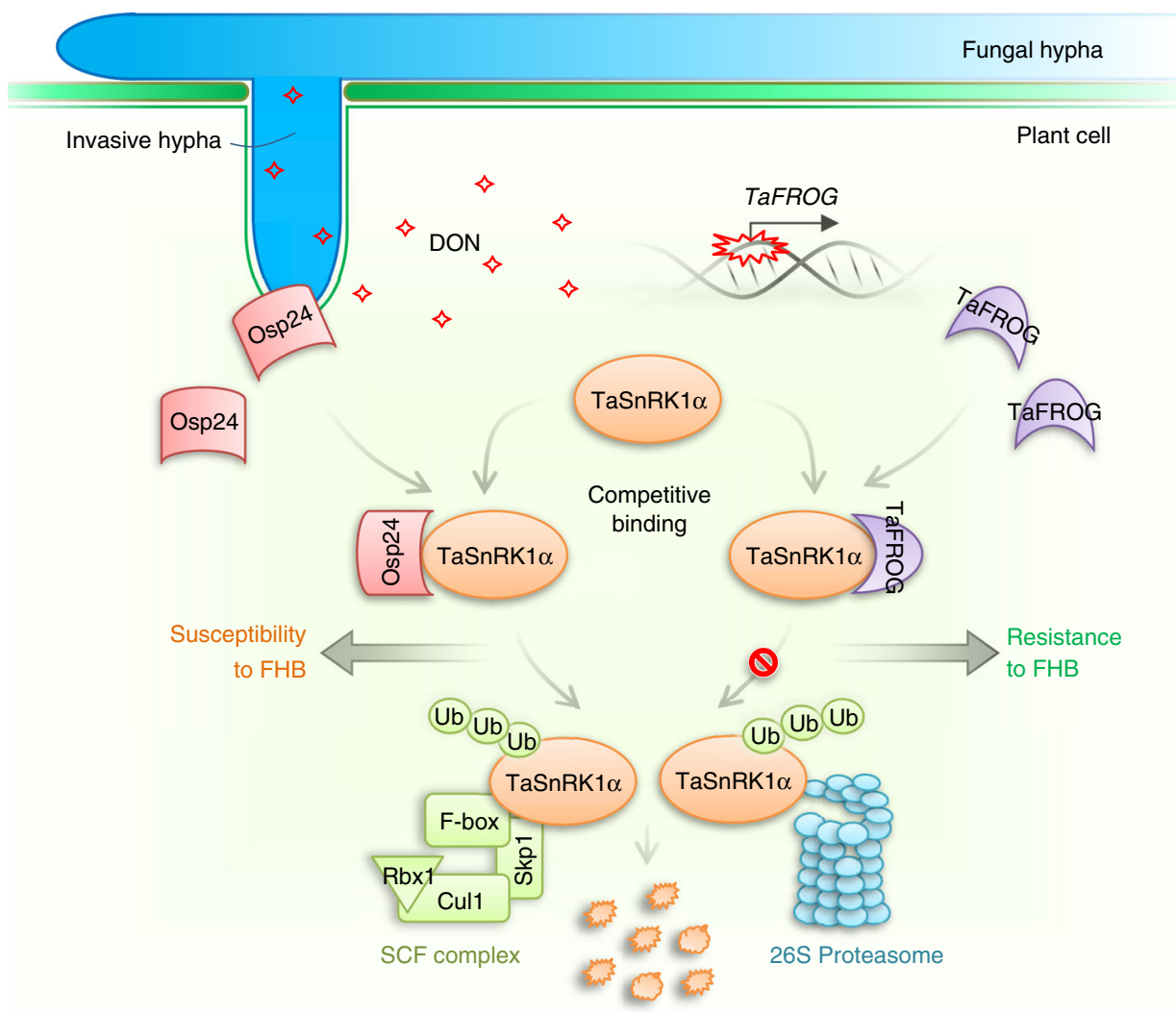

Fig. 5 A schematic summary of the roles of two orphan proteins from wheat and Fusarium graminearum during fungal-plant interactions. The cytoplasmic effector Osp24 secreted by invasive hyphae that develop in infected wheat tissues are translocated into plant cells. It interacts with TaSnRK1 $\alpha$, a protein kinase activated by DON and important for resistance against $F$. graminearum in wheat. The association of Osp24 with TaSnRK1 $\alpha$ stimulates its degradation by the SCF ubiquitin ligase complex and $26 \mathrm{~S}$ proteasome in infected plant cells, resulting in increased susceptibility to Fusarium head blight (FHB). However, DON produced by the fungal pathogen during infection induces the expression of TaFROG in wheat heads. TaFROG, a Pooideae-specific orphan protein, competes with Osp24 for binding with TaSnRK1 $\alpha$ and increases its stability. Protection by TaFROG from proteasome degradation and activation by DON enhance the functions of TaSnRK1 $\alpha$ in regulating defense responses against $F$. graminearum infection.

5-day-old liquid carboxymethyl cellulose (CMC) medium. For assaying defects in sexual reproduction, aerial hyphae of 5-day-old carrot agar cultures were pressed down with sterile $0.1 \%$ Tween 20 and then incubated at $25^{\circ} \mathrm{C}$ under black light. Perithecium formation was examined 7 days after induction for sexual reproduction ${ }^{17,72}$.

Gene replacement constructs were generated with the split-marker approach and transformed into protoplasts of PH-1 $1^{17,20}$. For each OSP gene, at least two independent gene replacement mutants were identified. For complementation assays, the entire OSP24 gene with its native promoter was cloned into plasmid pFL2 by gap repair ${ }^{42,43}$ and transformed into the osp 24 mutant. The osp $24 / O S P 24$ transformants were identified by PCR and assayed for phenotype complementation ${ }^{17}$. All the primers used in this study are described in Supplementary Data 3.

Infection assays with flowering wheat heads. Conidia were harvested from 5-day-old CMC cultures and re-suspended to $10^{5}$ spores $/ \mathrm{ml}$ in sterile distilled water (DDW). Flowering heads of 6-week-old wheat plants of cultivar Xiaoyan $22^{73}$, Zhoumai $36^{38}$, or KN199 were inoculated with $10 \mu \mathrm{l}$ of conidium suspensions at the fifth spikelet from the base ${ }^{72,74}$. Inoculated wheat heads were capped with a plastic bag for $48 \mathrm{~h}$ to keep the moisture. Infected wheat heads were examined for diseased spikelets at 7 or $14 \mathrm{dpi}$ to estimate the disease index (number of diseased spikeletsper head) ${ }^{75}$. Mean and standard deviation of the disease index were estimated with data from three independent replicates with at least 10 wheat heads examined in each replicate. DON production in the inoculated spikelets sampled at 14 dpi was assayed by GCMS-QP2010 system with AOC-20i autoinjector (Shimadzu Co. Japan). To assay infection cushion formation, infected lemmas were sampled at $2 \mathrm{dpi}$, fixed with $4 \%$ (vol/vol) glutaraldehyde, and coated with gold-palladium before examination with a JEOL 6360 scanning electron microscope (Jeol Ltd., Japan) $)^{14,20,76}$. For assaying infectious growth, infected rachis tissues were embedded in Spurr resin after fixation with 3\% glutaraldehyde and dehydration in graded series of $30-100 \%$ of ethanol before being sectioned ${ }^{77}$. Thick sections were then prepared and stained with $0.5 \%$ (wt/vol) toluidine blue before examination with an Olympus BX-53 microscope. Differences between the wildtype and mutant strains in infection cushion formation and infectious growth were determined with results from at least three independent replicates. Corn silks inoculated with culture blocks $(5 \mathrm{~mm})$ of $F$. graminearum were examined for discoloration at $7 \mathrm{dpi}^{78}$. For infection assays with tomatoes, each fruit was injected with $10 \mu \mathrm{l}$ of conidium suspensions after surface sterilization. Inoculated tomatoes were examined for tissue maceration after incubation at $25^{\circ} \mathrm{C}$ in dark for 7 days ${ }^{79}$. For infection assays with Arabidopsis, flowers were sprayed with conidium suspensions of $F$. graminearum $\left(10^{5}\right.$ spores $\left./ \mathrm{ml}\right)$. Inoculated plants were cultured in plastic propagators to keep the moisture and examined for necrosis in floral tissues at $7 \mathrm{dpi}^{80}$.

RNA-seq analysis. The RNA-seq reads of wheat heads infected with PH-1 and osp 24 mutant were mapped to the reference genome of PH-1 via hisat $2^{81}$. The expression level of each gene was counted with featureCounts ${ }^{82}$. DEGs were identified by edgeRun ${ }^{83}$. Functional Catalog (FunCat) ${ }^{84}$ and GO-enrichment analyses were performed with custom script (https://github.com/xulab-nwafu/ funcat) and Blast $2 \mathrm{GO}^{85}$, respectively.

Assays for the function of the SP of Osp24. The predicted SP (22 aa) of Osp24 $\left(\mathrm{SP}^{22}\right)$ was cloned into the pSUC2 vector ${ }^{86}$ that carries the yeast SUC2 gene deleted of its SP sequence. The resulting $\mathrm{SP}^{22}-S U C 2$ construct was transformed into the yeast suc2 mutant YTK $12^{87}$ and assayed for growth on CMD-W $(0.67 \%$ yeast nitrogen base without AAs, $0.075 \%$ tryptophan dropout supplement, $2 \%$ sucrose, $0.1 \%$ glucose, and $2 \%$ agar) and YPRAA medium plate (1\% yeast extract, $2 \%$ peptone, $2 \%$ raffinose, and $2 \mu \mathrm{g} / \mathrm{ml}$ antimicyn A $)^{88}$. Transformants of YTK12 carrying the empty pSUC vector or pSUC2-Avr1b ${ }^{\text {SP89 }}$ were used as the negative and positive controls, respectively. The invertase enzymatic activity was detected by the reduction of 2,3,5-triphenyltetrazolium chloride (TTC) to insoluble red colored 1,3,5-triphenylformazan (TPF).

Assays for the suppression of BAX-induced or INF1-induced cell death by Osp24. $\mathrm{P}_{\mathrm{CaMV}} 35 \mathrm{~S}^{-} \mathrm{Osp} 24^{\Delta \mathrm{SP}}$ was cloned into pGR106 and transformed into A. tumefaciens strain GV310190 expressing the BAX and INF1 constructs ${ }^{91}$. For infiltration assays with $N$. benthamiana leaves, A. tumefaciens cells were 
resuspended to $\mathrm{OD}_{600}$ of 0.8 in infiltration solution (10 mM MES, $10 \mathrm{mM} \mathrm{MgCl}_{2}$, and $150 \mu \mathrm{M}$ acetosyringone $)^{90}$. At $18 \mathrm{~h}$ after the initial infiltration of tobacco leaves with $A$. tumefaciens transformant carrying the $\mathrm{P}_{\mathrm{CaMV}}{ }_{35 \mathrm{~S}} \mathrm{O}$ Osp24${ }^{\Delta \mathrm{SP}}$ construct, the same sites were infiltrated with cells carrying GFP, BAX, or INF1 constructs ${ }^{40}$. Plant cell death symptoms were examined 5 days after infiltration with cells expressing BAX or INF1. A. tumefaciens cells carrying the GFP vector ${ }^{40}$ were used as the negative control. Each infiltration experiment was repeated at least three times with a minimum of three leaves tested. The expression of BAX and Osp24 in $N$. benthamiana leaves were assayed by western blot analysis with the anti-BAX and anti-GFP antibodies.

Assays for the localization of Osp24 during plant infection. The Osp24mCherry-NLS fusion construct under the control of its native promoter was generated by overlapping PCR with the NLS sequence from simian virus large Tantigen ${ }^{92}$ and transformed into the wild-type stain PH-1. Conidium suspensions $\left(10^{5}\right.$ spores $\left./ \mathrm{ml}\right)$ of the resulting transformants were used for infection assays with wheat seedlings ${ }^{15}$. Infectious hyphae and mCherry signals in plant tissues were examined at 2 dpi with a Nikon A1 microscope at excitation/emission wavelengths $543 \mathrm{~nm} / 560-615 \mathrm{~nm}$.

Yeast two-hybrid assays. For library construction, RNA was isolated from wheat heads of Xiaoyan 22 inoculated with PH-1 and sampled at 3 dpi. The yeast twohybrid library of 6 million primary clones was constructed with vector pGADT7 (Takara Bio, Japan) by OEBiotech (Shanghai, China). For library screening, the Osp24 ${ }^{\triangle S P}$ bait construct was generated with vector pGBKT7 (Takara Bio, Japan). Yeast colonies that grew on SD-Trp-Leu-His-Ade and had $\beta$-galactosidase activity were isolated as putative OICs. After sequencing with primer T7, wheat genes corresponding to the inserts in these clones were identified by Blast searches. To directly assay their interactions, full-length cDNAs or fragments of TaSnRK1a, Osp24, and TaFROG were amplified and cloned into pGADT7 or pGBKT7. The resulting bait and prey constructs of TaSnRK1 $\alpha$, Osp24, and TaFROG were transformed in pairs into yeast strain $\mathrm{AH} 109$. The $\mathrm{Leu}^{+}$and $\mathrm{Trp}^{+}$transformants were isolated and assayed for growth on SD-Trp-Leu-His medium and galactosidase activities in filter lift assays ${ }^{78}$

BiFC assays for the TaSnRK1 $\alpha-0 s p 24$ interaction. The TaSnRK1 $\alpha$ and Osp24 ${ }^{\triangle S P}$ fragments were cloned into the BiFC vectors pSPYNE-35S and pSPYCE$35 S^{93}$, respectively. The resulting Osp24-nYFP and TaSnRK1a-cYFP constructs were transformed into A. tumefaciens strain GV3101. Leaves of $N$. benthamiana were then infiltrated with Agrobacterium cells expressing Osp24-nYFP and/or TaSnRK1 $\alpha$-cYFP as described above ${ }^{91}$. Two-days after infiltration, fluorescence signals were examined with a Nikon A1 microscope. Nuclei were stained with 4,6diamidino-2-phenylindole (DAPI).

In vitro pull-down assays. Osp24, TaSnRK1 $\alpha$, and TaFROG cDNA fragments were amplified and cloned into pGEX4T $1^{94}$ and pCold ${ }^{95}$ for purification of Osp24GST, TaSnRK1a-His, and TaFROG-S recombinant proteins $s^{96}$. To assay their interactions, equal amounts of Osp24-GST and TaSnRK1a-HIS fusion proteins were incubated at $4{ }^{\circ} \mathrm{C}$ for $2 \mathrm{~h}$ and mixed with glutathione or Ni-NTA resins (GenScript, China) for affinity purification ${ }^{97,98}$. The presence of Osp24-GST and TaSnRK1a-HIS in proteins eluted from Ni-NTA resins was detected by western blot analysis with the anti-HIS (1:5000 dilution, CW0286, CoWin Bioscience Co., China) and anti-GST (1:5000 dilution, CW0084, CoWin Bioscience Co., China) antibodies. For assaying competition between Osp24 and TaFROG, equal amounts of Osp24-GST and TaSnRK1 $\alpha$-HIS fusion proteins were co-incubated with varying amounts of TaFROG-S-tag proteins $(1 \times, 2 \times$, and $4 \times$ ) before mixing with Ni-NTA resins. To assay the interaction between TaSnRK1a and the ubiquitin-26S proteasome system, total proteins were isolated from wheat heads ${ }^{20,78}$ and coincubated with TaSnRK1 $\alpha$-HIS for 30 min before mixing with Ni-NTA resins in the presence of $50 \mu \mathrm{M}$ proteasome inhibitor MG132 $2^{67}$. Western blots of proteins eluted from the Ni-NTA resins were detected with the anti-RBX1 (1:1000 dilution, ab133565, Abcam, UK) and anti-20s (1:1000 dilution, ab22674, Abcam, UK) antibodies for the presence of RBX1 protein and 20S proteasome ${ }^{37}$.

Cell-free protein degradation assays. Total proteins isolated from wheat heads $s^{20,78}$ and TaSnRK1 $\alpha$-HIS recombinant proteins were used for the cell-free protein degradation assays ${ }^{99,100}$. In brief, a final concentration of $10 \mathrm{mM}$ ATP was added to the mixture of equal amounts of TaSnRK1a-HIS and crude protein extract isolated from infected wheat heads ${ }^{100}$. Detection with an anti-actin antibody (1:1000 dilution, BE0027, Easybio, China) is used as a loading control. To inhibit the 26S proteasome activity, a final concentration of $50 \mu \mathrm{M}$ MG132 was added to the reaction mixtures ${ }^{67}$. The degradation reaction was stopped after incubation at $25^{\circ} \mathrm{C}$ for 0,45 , and $90 \mathrm{~min}$ by boiling for $5 \mathrm{~min}$ in SDS sample buffer. Western blots of these reaction mixtures were detected for the remaining TaSnRK1a-HIS proteins with an anti-HIS antibody (1:5000 dilution, CW0286, CoWin Bioscience Co., China). Each experiment was repeated at least three times.
Generation of transgenic wheat plants. For overexpression, the full-length TaSnRKla ORF was cloned into the pANIC-5E vector behind the $35 \mathrm{~S}$ promoter ${ }^{101}$. For silencing, a 107-bp fragment of TaSnRK1a was amplified and cloned into vector pANIC-7E in both antisense and sense orientations by Gateway cloning ${ }^{101}$. The resulting constructs were transformed into immature embryos of wheat cultivar KN199 by particle bombardment ${ }^{32}$ at Genovo Bio (Tianjin, China). Transgenic plants resistant to BASTA were verified by PCR for carrying transforming constructs with DNA isolated from leaves of T0 plants. The expression level of TaSnRK1a was assayed by qRT-PCR with RNA isolated from wheat heads of selected T1 and T2 plants. Transgenic lines with over 2fold increase or reduction of TaSnRK1a expression were selected for infection assays with $F$. graminearum as described above ${ }^{20}$. Similar approaches were used to generate TaFROG overexpression constructs and transgenic plants. To quantify fungal biomass in infected plant tissues, genomic DNA was extracted from infected wheat heads sampled at $5 \mathrm{dpi}$ and used for $\mathrm{QPCR}$ assays with primers specific for the wheat GAPDH ${ }^{102}$ and F. graminearum CHS5 genes ${ }^{103}$. Results from three independent biological replicates were used to estimate the ratio of wheat and F. graminearum biomasses.

Reporting summary. Further information on research design is available in the Nature Research Reporting Summary linked to this article.

\section{Data availability}

Data supporting the major findings of this work are available within the paper and its Supplementary Information files. RNA-seq data were uploaded to the NCBI Sequence Read Archive under the accessions SRR12342821-SRR12342826. Any other supporting data is available from the corresponding authors upon request. Source data are provided with this paper.

\section{Code availability}

The custom script for FunCat enrichment analysis is available from GitHub (https:// github.com/xulab-nwafu/funcat).

Received: 11 February 2020; Accepted: 6 August 2020; Published online: 01 September 2020

\section{References}

1. Plissonneau, C., Sturchler, A. \& Croll, D. The evolution of orphan regions in genomes of a fungal pathogen of wheat. MBio 7, e01231-01216 (2016).

2. Domazet-Loso, T. \& Tautz, D. An evolutionary analysis of orphan genes in Drosophila. Genome Res. 13, 2213-2219 (2003).

3. Wissler, L., Gadau, J., Simola, D. F., Helmkampf, M. \& Bornberg-Bauer, E. Mechanisms and dynamics of orphan gene emergence in insect genomes. Genome Biol. Evol. 5, 439-455 (2013).

4. Giraldo, M. C. \& Valent, B. Filamentous plant pathogen effectors in action. Nat. Rev. Microbiol. 11, 800-814 (2013).

5. Stergiopoulos, I. \& de Wit, P. J. Fungal effector proteins. Annu. Rev. Phytopathol. 47, 233-263 (2009).

6. Khang, C. H. et al. Translocation of Magnaporthe oryzae effectors into rice cells and their subsequent cell-to-cell movement. Plant Cell 22, 1388-1403 (2010)

7. Skibbe, D. S., Doehlemann, G., Fernandes, J. \& Walbot, V. Maize tumors caused by Ustilago maydis require organ-specific genes in host and pathogen. Science 328, 89-92 (2010).

8. Qi, T. et al. Stripe rust effector PstGSRE1 disrupts nuclear localization of ROS promoting transcription factor TaLOL2 to defeat ROS-induced defense in wheat. Mol. Plant 12, 1624-1638 (2019).

9. Shen, Q., Liu, Y. Y. \& Naqvi, N. I. Fungal effectors at the crossroads of phytohormone signaling. Curr. Opin. Microbiol. 46, 1-6 (2018).

10. Selin, C., de Kievit, T. R., Belmonte, M. F. \& Fernando, W. G. D. Elucidating the role of effectors in plant-fungal interactions: progress and challenges. Front. Microbiol. 7, 110 (2016).

11. Tanaka, S. et al. Neofunctionalization of the secreted Tin 2 effector in the fungal pathogen Ustilago maydis. Nat. Microbiol. 4, 251-257 (2019).

12. Tanaka, S. et al. A secreted Ustilago maydis effector promotes virulence by targeting anthocyanin biosynthesis in maize. Elife 3, e01355 (2014).

13. Djamei, A. et al. Metabolic priming by a secreted fungal effector. Nature $\mathbf{4 7 8}$, 395-398 (2011).

14. Boenisch, M. J. \& Schafer, W. Fusarium graminearum forms mycotoxin producing infection structures on wheat. BMC Plant Biol. 11, 110 (2011).

15. Zhang, X. W. et al. In planta stage-specific fungal gene profiling elucidates the molecular strategies of Fusarium graminearum growing inside wheat coleoptiles. Plant Cell 24, 5159-5176 (2012). 
16. Rittenour, W. R. \& Harris, S. D. An in vitro method for the analysis of infection-related morphogenesis in Fusarium graminearum. Mol. Plant Pathol. 11, 361-369 (2010).

17. Wang, C. F. et al. Functional analysis of the kinome of the wheat scab fungus Fusarium graminearum. PLoS Pathog. 7, e1002460 (2011).

18. Yun, Y. et al. Functional analysis of the Fusarium graminearum phosphatome. New Phytol. 207, 119-134 (2015).

19. Son, H. et al. A phenome-based functional analysis of transcription factors in the cereal head blight fungus, Fusarium graminearum. PLoS Pathog. 7 , e1002310 (2011).

20. Jiang, C. et al. An expanded subfamily of G-protein-coupled receptor genes in Fusarium graminearum required for wheat infection. Nat. Microbiol. 4, 1582-1591 (2019).

21. Voigt, C. A., Schafer, W. \& Salomon, S. A secreted lipase of Fusarium graminearum is a virulence factor required for infection of cereals. Plant J. 42, 364-375 (2005).

22. Qi, P. F. et al. Functional analysis of FgNahG clarifies the contribution of salicylic acid to wheat (Triticum aestivum) resistance against Fusarium head blight. Toxins 11, E59 (2019).

23. Jia, L. J. et al. A linear nonribosomal octapeptide from Fusarium graminearum facilitates cell-to-cell invasion of wheat. Nat. Commun. 10, 922 (2019).

24. Audenaert, K., Vanheule, A., Hofte, M. \& Haesaert, G. Deoxynivalenol: a major player in the multifaceted response of Fusarium to its environment. Toxins 6, 1-19 (2013).

25. Van de Walle, J. et al. Deoxynivalenol affects in vitro intestinal epithelial cell barrier integrity through inhibition of protein synthesis. Toxicol. Appl. Pharm. 245, 291-298 (2010).

26. Jansen, C. et al. Infection patterns in barley and wheat spikes inoculated with wild-type and trichodiene synthase gene disrupted Fusarium graminearum. Proc. Natl Acad. Sci. USA 102, 16892-16897 (2005).

27. Shin, S. et al. Transgenic Arabidopsis thaliana expressing a barley UDPglucosyltransferase exhibit resistance to the mycotoxin deoxynivalenol. J. Exp. Bot. 63, 4731-4740 (2012).

28. Perochon, A. et al. TaFROG encodes a pooideae orphan protein that interacts with SnRK1 and enhances resistance to the mycotoxigenic fungus Fusarium graminearum. Plant Physiol. 169, 2895-2906 (2015).

29. Perochon, A. et al. A wheat NAC interacts with an orphan protein and enhances resistance to Fusarium head blight disease. Plant Biotechnol. J. 17, 1892-1904 (2019).

30. Rawat, N. et al. Wheat Fhb1 encodes a chimeric lectin with agglutinin domains and a pore-forming toxin-like domain conferring resistance to Fusarium head blight. Nat. Genet. 48, 1576-1580 (2016).

31. Su, Z. et al. A deletion mutation in TaHRC confers Fhbl resistance to Fusarium head blight in wheat. Nat. Genet. 51, 1099-1105 (2019).

32. Li, G. et al. Mutation of a histidine-rich calcium-binding-protein gene in wheat confers resistance to Fusarium head blight. Nat. Genet. 51, 1106-1112 (2019).

33. Cuomo, C. A. et al. The Fusarium graminearum genome reveals a link between localized polymorphism and pathogen specialization. Science 317, 1400-1402 (2007).

34. Zhang, S. \& Xu, J. R. Effectors and effector delivery in Magnaporthe oryzae. PLoS Pathog. 10, e1003826 (2014).

35. Koeck, M., Hardham, A. R. \& Dodds, P. N. The role of effectors of biotrophic and hemibiotrophic fungi in infection. Cell. Microbiol. 13, 1849-1857 (2011).

36. Brown, N. A., Antoniw, J. \& Hammond-Kosack, K. E. The predicted secretome of the plant pathogenic fungus Fusarium graminearum: a refined comparative analysis. PLoS ONE 7, e33731 (2012).

37. Hilt, W. \& Wolf, D. H. The ubiquitin-proteasome system: past, present and future. Cell. Mol. Life Sci. 61, 1545 (2004).

38. Li, T. et al. Effects of the Fhbl gene on Fusarium head blight resistance and agronomic traits of winter wheat. Crop J. 7, 799-808 (2019).

39. Peng, Z. et al. Effector gene reshuffling involves dispensable minichromosomes in the wheat blast fungus. PLoS Genet. 15, e1008272 (2019).

40. Zhao, M. et al. Candidate effector Pst_8713 impairs the plant immunity and contributes to virulence of Puccinia striiformis f. sp. tritici. Front. Plant Sci. 9, 1294 (2018).

41. Gietz, R. D., Schiestl, R. H., Willems, A. R. \& Woods, R. A. Studies on the transformation of intact yeast-cells by the Liac/S-DNA/Peg procedure. Yeast 11, 355-360 (1995).

42. Zhou, X., Li, G. \& Xu, J. R. Efficient approaches for generating GFP fusion and epitope-tagging constructs in filamentous fungi. Methods Mol. Biol. 722, 199-212 (2011).

43. Bruno, K. S., Tenjo, F., Li, L., Hamer, J. E. \& Xu, J. R. Cellular localization and role of kinase activity of PMK1 in Magnaporthe grisea. Eukaryot. Cell 3, 1525-1532 (2004).

44. Lin, H. H. et al. Disulfide connectivity prediction based on structural information without a prior knowledge of the bonding state of cysteines. Comput. Biol. Med. 43, 1941-1948 (2013).
45. Zhao, G. P. et al. Activation of the proapoptotic Bcl-2 protein bax by a small molecule induces tumor cell apoptosis. Mol. Cell. Biol. 34, 1198-1207 (2014).

46. Kamoun, S. et al. A gene encoding a protein elicitor of Phytophthora infestans is down-regulated during infection of potato. Mol. Plant Microbe 10, 13-20 (1997).

47. McHale, L., Tan, X. P., Koehl, P. \& Michelmore, R. W. Plant NBS-LRR proteins: adaptable guards. Genome Biol. 7, 212 (2006).

48. Jossier, M. et al. SnRK1 (SNF1-related kinase 1) has a central role in sugar and ABA signalling in Arabidopsis thaliana. Plant J. 59, 316-328 (2009).

49. Takahashi, A., Casais, C., Ichimura, K. \& Shirasu, K. HSP90 interacts with RAR1 and SGT1 and is essential for RPS2-mediated disease resistance in Arabidopsis. Proc. Natl Acad. Sci. USA 100, 11777-11782 (2003).

50. Perochon, A. et al. The wheat SnRK1alpha family and its contribution to Fusarium toxin tolerance. Plant Sci. 288, 110217 (2019).

51. Zou, S. H., Wang, H., Li, Y. W., Kong, Z. S. \& Tang, D. Z. The NB-LRR gene Pm60 confers powdery mildew resistance in wheat. New Phytol. 218, 298-309 (2018).

52. Pauli, S., Rothnie, H. M., Gang, C., He, X. Y. \& Hohn, T. The cauliflower mosaic virus $35 \mathrm{~S}$ promoter extends into the transcribed region. J. Virol. 78, 12120-12128 (2004).

53. Farras, R. et al. SKP1-SnRK protein kinase interactions mediate proteasomal binding of a plant SCF ubiquitin ligase. Embo J. 20, 2742-2756 (2001).

54. Bard, J. A. M. et al. Structure and function of the $26 \mathrm{~S}$ proteasome. Annu. Rev. Biochem. 87, 697-724 (2018).

55. Wang, Q. H. et al. Characterization of the two-speed subgenomes of Fusarium graminearum reveals the fast-speed subgenome specialized for adaption and infection. Front. Plant Sci. 8, 140 (2017).

56. Ma, L. J. \& Xu, J. R. Shuffling effector genes through mini-chromosomes. PLoS Genet. 15, e1008345 (2019).

57. Lyu, X. L. et al. A small secreted virulence-related protein is essential for the necrotrophic interactions of Sclerotinia sclerotiorum with its host plants. PLoS Pathog. 12, e1005435 (2016).

58. Liu, Z. et al. The cysteine rich necrotrophic effector SnToxl produced by Stagonospora nodorum triggers susceptibility of wheat lines harboring Snn1. PLoS Pathog. 8, e1002467 (2012).

59. Emanuelle, S., Doblin, M. S., Stapleton, D. I., Bacic, A. \& Gooley, P. R. Molecular insights into the enigmatic metabolic regulator, SnRK1. Trends Plant Sci. 21, 341-353 (2016).

60. Seo, Y. S. et al. Towards establishment of a rice stress response interactome. PLoS Genet. 7, e1002020 (2011).

61. Shen, W., Dallas, M. B., Goshe, M. B. \& Hanley-Bowdoin, L. SnRK1 phosphorylation of AL2 delays cabbage leaf curl virus infection in Arabidopsis. J. Virol. 88, 10598-10612 (2014).

62. Szczesny, R. et al. Suppression of the AvrBs1-specific hypersensitive response by the YopJ effector homolog AvrBsT from Xanthomonas depends on a SNF1-related kinase. New Phytol. 187, 1058-1074 (2010).

63. Hulsmans, S., Rodriguez, M., De Coninck, B. \& Rolland, F. The SnRK1 energy sensor in plant biotic interactions. Trends Plant Sci. 21, 648-661 (2016).

64. Filipe, O., De Vleesschauwer, D., Haeck, A., Demeestere, K. \& Hofte, M. The energy sensor OsSnRK1a confers broad-spectrum disease resistance in rice. Sci. Rep. 8, 3864 (2018).

65. Kim, C. Y., Vo, K. T. X., An, G. \& Jeon, J. S. A rice sucrose non-fermenting-1 related protein kinase 1, OSK35, plays an important role in fungal and bacterial disease resistance. J. Korean Soc. Appl Biol. Chem. 58, 669-675 (2015).

66. Lu, Z. \& Hunter, T. Degradation of activated protein kinases by ubiquitination. Annu. Rev. Biochem. 78, 435-475 (2009).

67. Chen, G. et al. MG132 proteasome inhibitor upregulates the expression of connexin 43 in rats with adriamycin-induced heart failure. Mol. Med. Rep. 12, 7595-7602 (2015).

68. Mueller, A. N., Ziemann, S., Treitschke, S., Assmann, D. \& Doehlemann, G. Compatibility in the Ustilago maydis-Maize interaction requires inhibition of host cysteine proteases by the fungal effector Pit2. PLoS Pathog. 9, e1003177 (2013).

69. van Esse, H. P. et al. The Cladosporium fulvum virulence protein Avr2 inhibits host proteases required for basal defense. Plant Cell 20, 1948-1963 (2008).

70. Park, C. H. et al. The Magnaporthe oryzae effector AvrPiz-t targets the RING E3 ubiquitin ligase APIP6 to suppress pathogen-associated molecular patterntriggered immunity in rice. Plant Cell 24, 4748-4762 (2012).

71. Cuzick, A., Urban, M. \& Hammond-Kosack, K. Fusarium graminearum gene deletion mutants mapl and tri5 reveal similarities and differences in the pathogenicity requirements to cause disease on Arabidopsis and wheat floral tissue. New Phytol. 177, 990-1000 (2008)

72. Hou, Z. M. et al. A mitogen-activated protein kinase gene (MGV1) in Fusarium graminearum is required for female fertility, heterokaryon formation, and plant infection. Mol. Plant Microbe 15, 1119-1127 (2002).

73. Kang, Z. S., Buchenauer, H., Huang, L. L., Han, Q. M. \& Zhang, H. C. Cytological and immunocytochemical studies on responses of wheat spikes of 
the resistant Chinese cv. Sumai 3 and the susceptible cv. Xiaoyan 22 to infection by Fusarium graminearum. Eur. J. Plant Pathol. 120, 383-396 (2008).

74. Ding, S. L. et al. Transducin beta-like gene FTL1 is essential for pathogenesis in Fusarium graminearum. Eukaryot. Cell 8, 867-876 (2009).

75. Jonkers, W., Dong, Y. H., Broz, K. \& Kistler, H. C. The Worl-like protein Fgp1 regulates pathogenicity, toxin synthesis and reproduction in the phytopathogenic fungus Fusarium graminearum. PLoS Pathog. 8, e1002724 (2012).

76. $\mathrm{Hu}, \mathrm{S}$. et al. The cAMP-PKA pathway regulates growth, sexual and asexual differentiation, and pathogenesis in Fusarium graminearum. Mol. Plant Microbe 27, 557-566 (2014).

77. Yin, T. et al. The cyclase-associated protein FgCap1 has both protein kinase Adependent and -independent functions during deoxynivalenol production and plant infection in Fusarium graminearum. Mol. Plant Pathol. 19, 552-563 (2018).

78. Liu, H. et al. Two Cdc2 kinase genes with distinct functions in vegetative and infectious hyphae in Fusarium graminearum. PLoS Pathog. 11, e1004913 (2015).

79. Gu, Q., Chen, Y., Liu, Y., Zhang, C. Q. \& Ma, Z. H. The transmembrane protein FgShol regulates fungal development and pathogenicity via the MAPK module Ste50-Ste11-Ste7 in Fusarium graminearum. New Phytol. 206, 315-328 (2015).

80. Urban, M., Daniels, S., Mott, E. \& Hammond-Kosack, K. Arabidopsis is susceptible to the cereal ear blight fungal pathogens Fusarium graminearum and Fusarium culmorum. Plant J. 32, 961-973 (2002).

81. Kim, D., Langmead, B. \& Salzberg, S. L. HISAT: a fast spliced aligner with low memory requirements. Nat. Methods 12, 357-360 (2015)

82. Liao, Y., Smyth, G. K. \& Shi, W. featureCounts: an efficient general purpose program for assigning sequence reads to genomic features. Bioinformatics $\mathbf{3 0}$, 923-930 (2014).

83. Dimont, E., Shi, J., Kirchner, R. \& Hide, W. edgeRun: an R package for sensitive, functionally relevant differential expression discovery using an unconditional exact test. Bioinformatics 31, 2589-2590 (2015).

84. Ruepp, A. et al. The FunCat, a functional annotation scheme for systematic classification of proteins from whole genomes. Nucleic Acids Res. 32, 5539-5545 (2004).

85. Conesa, A. et al. Blast2GO: a universal tool for annotation, visualization and analysis in functional genomics research. Bioinformatics 21, 3674-3676 (2005).

86. Jacobs, K. A. et al. A genetic selection for isolating cDNAs encoding secreted proteins. Gene 198, 289-296 (1997).

87. Oh, S. K. et al. In planta expression screens of Phytophthora infestans RXLR effectors reveal diverse phenotypes, including activation of the Solanum bulbocastanum disease resistance protein Rpi-blb2. Plant Cell 21, 2928-2947 (2009).

88. Koschwanez, J. H., Foster, K. R. \& Murray, A. W. Sucrose utilization in budding yeast as a model for the origin of undifferentiated multicellularity. PLoS Biol. 9, e1001122 (2011).

89. Dou, D. et al. RXLR-mediated entry of Phytophthora sojae effector Avr1b into soybean cells does not require pathogen-encoded machinery. Plant Cell 20, 1930-1947 (2008).

90. Han, Z. F., Hunter, D. M., Sibbald, S., Zhang, J. S. \& Tian, L. Biological activity of the tzs gene of nopaline Agrobacterium tumefaciens GV3101 in plant regeneration and genetic transformation. Mol. Plant-Microbe Interact. 26 1359-1365 (2013).

91. Xu, Q. et al. An effector protein of the wheat stripe rust fungus targets chloroplasts and suppresses chloroplast function. Nat. Commun. 10, 5571 (2019).

92. Ai, H. W., Shaner, N. C., Cheng, Z., Tsien, R. Y. \& Campbell, R. E. Exploration of new chromophore structures leads to the identification of improved blue fluorescent proteins. Biochemistry 46, 5904-5910 (2007).

93. Walter, M. et al. Visualization of protein interactions in living plant cells using bimolecular fluorescence complementation. Plant J. 40, 428-438 (2004).

94. Shen, M. et al. Identification of glutathione S-transferase (GST) genes from a dark septate endophytic fungus (Exophiala pisciphila) and their expression patterns under varied metals stress. PLOS ONE 10, e0123418 (2015).

95. Qing, G. et al. Cold-shock induced high-yield protein production in Escherichia coli. Nat. Biotechnol. 22, 877-882 (2004).

96. Peleg, Y. \& Unger, T. Resolving bottlenecks for recombinant protein expression in E. coli. Methods Mol. Biol. 800, 173-186 (2012).
97. Swaffield, J. C. \& Johnston, S. A. Affinity purification of proteins binding to GST fusion proteins. Curr. Protoc. Mol. Biol. Chapter 20, Unit 20 22 (2001).

98. Kong, L. et al. A Phytophthora effector manipulates host histone acetylation and reprograms defense gene expression to promote infection. Curr. Biol. 27, 981-991 (2017).

99. Wang, F. et al. Biochemical insights on degradation of Arabidopsis DELLA proteins gained from a cell-free assay system. Plant Cell 21, 2378-2390 (2009)

100. Kong, L. et al. Degradation of the ABA co-receptor ABI1 by PUB12/13 U-box E3 ligases. Nat. Commun. 6, 8630 (2015).

101. Mann, D. G. et al. Gateway-compatible vectors for high-throughput gene functional analysis in switchgrass (Panicum virgatum L.) and other monocot species. Plant Biotechnol. J. 10, 226-236 (2012).

102. Zeng, L., Deng, R., Guo, Z., Yang, S. \& Deng, X. Genome-wide identification and characterization of Glyceraldehyde-3-phosphate dehydrogenase genes family in wheat (Triticum aestivum). BMC Genomics 17, 240 (2016).

103. Li, H. et al. A Phytophthora effector recruits a host cytoplasmic transacetylase into nuclear speckles to enhance plant susceptibility. Elife 7, e40039 (2018).

\section{Acknowledgements}

We thank Zhe Tang, Ping Xiang, Jiangang Kang, Gang Niu, and Guoyun Zhang for technical supports, Drs. Xue Zhang and Guanghui Wang for fruitful discussions, and Dr. Larry Dunkle at Purdue University for critical reading of this manuscript. We also thank Drs. Zhensheng Kang and Xiaojie Wang at Northwest A\&F University for providing plasmids used in this study. This work was supported by grants from National Natural Science Foundation of China (Nos. 31671981, 31601587, and 31701747), New Star of Youth Science and Technology of Shaanxi Province (2018KJXX-068), Tang Scholar and USWBSI.

\section{Author contributions}

C.J., J.-R.X., and H.Q.L. designed the experiments and wrote the paper. C.J., R.N.H., Y.Y., S.J.Z., Q.Z., W.W., M.Y., G.R.Z., and P.P.H. performed the experiments. C.J., H.Q.L., and Q.H.W. analyzed the data.

\section{Competing interests}

The authors declare no competing interests.

\section{Additional information}

Supplementary information is available for this paper at https://doi.org/10.1038/s41467020-18240-y.

Correspondence and requests for materials should be addressed to H.L. or J.-R.X.

Peer review information Nature Communications thanks Frederik Börnke, Donald Gardiner, and the other, anonymous reviewer(s) for their contribution to the peer review of this work. Peer review reports are available

Reprints and permission information is available at http://www.nature.com/reprints

Publisher's note Springer Nature remains neutral with regard to jurisdictional claims in published maps and institutional affiliations.

Open Access This article is licensed under a Creative Commons Attribution 4.0 International License, which permits use, sharing, adaptation, distribution and reproduction in any medium or format, as long as you give appropriate credit to the original author(s) and the source, provide a link to the Creative Commons license, and indicate if changes were made. The images or other third party material in this article are included in the article's Creative Commons license, unless indicated otherwise in a credit line to the material. If material is not included in the article's Creative Commons license and your intended use is not permitted by statutory regulation or exceeds the permitted use, you will need to obtain permission directly from the copyright holder. To view a copy of this license, visit http://creativecommons.org/ licenses/by/4.0/

(C) The Author(s) 2020 